\title{
Do We Really Want to Keep the Gate Threshold That High?
}

\author{
GRACE BROOKS, ${ }^{1,2}$ AES Student Member, AMANDINE PRAS, ${ }^{3,4,1}$ AES Member, \\ (Grace.Brooks@mail.mcgill.ca) \\ (Amandine.Pras@uleth.ca) \\ ATHENA ELAFROS, ${ }^{5}$ AND MONICA LOCKETT ${ }^{5}$ \\ (Athena.Elafros@uleth.ca) (M.Lockett@uleth.ca)
${ }^{1}$ Centre for Interdisciplinary Research in Music Media and Technology (CIRMMT), Montreal, Quebec
${ }^{2}$ School of Information Studies, McGill University, Montreal, Quebec
${ }^{3}$ Digital Audio Arts program, Department of Music, University of Lethbridge, Lethbridge, Alberta
${ }^{4}$ Centre George Simmel, School of Advanced Studies in the Social Sciences (EHESS), Paris, France \\ ${ }^{5}$ Department of Sociology, University of Lethbridge, Lethbridge, Alberta
}

\begin{abstract}
Drawing upon the survey instruments of Lewis and Neville [1], Nadal [2], and Yang and Carroll [3], we conducted an online survey that captured experiences of discrimination and microaggressions reported by 387 recording engineers, producers, and studio assistants living in 46 different countries. Our statistical analyses reveal highly significant and systemic gender inequalities within the field, e.g., cisgender women experience many more sexually inappropriate comments ( $p<\mathrm{e}-14$, large effect size) and unwanted comments about their physical appearance ( $p<\mathrm{e}-12$, large effect size) than cisgender men, and they are much more likely to face challenges to their authority ( $p<\mathrm{e}-13$, large effect size) and expertise $(p<\mathrm{e}-10$, large effect size). A comparison of our results with a study about women's experiences of microaggressions within STEM academia [3] indicates that the recording studio workplace scores 33\% worse on the silencing and marginalization of women, $33 \%$ worse on gender-related workplace microaggressions, and $24 \%$ worse on sexual objectification. These findings call for serious reflection on the part of the community to progress from awareness to collective action that will unlock the control room for women and other historically and systemically marginalized groups of studio professionals.
\end{abstract}

\section{INTRODUCTION}

In a podcast entitled The Midwife of Audio, ${ }^{1}$ mixing engineer Tom Elmhirst defines his role as a facilitator in the process of music delivery that is audio production. This title obviously invokes associations with gender, birth, and social reproduction. Yet women comprise less than $5 \%$ of audio engineers according to the Women's Audio Mission [4] and between 5\% and 10\% of audio engineers according to AES [4]. A recent study investigating gender composition by presentation type at AES Conventions from 2012-2019 shows that very few women and non-binary authors are represented in Invited Papers (1.96\%), Keynotes (8.51\%), and Workshops (9.73\%) [6]. Also, an Annenberg study showed that only $2 \%$ of the record producers credited on 20122017 Billboard top 100 hits are women, only $0.3 \%$ of these producers ( 2 out of 651) are women from underrepresented racial/ethnic groups, and no woman producer was Grammy nominated from 2013-2018 [7].

To challenge these harsh gender inequalities (among other social discrimination and equity issues), the AES created a Diversity \& Inclusion (D\&I) Committee in Oct. 2017 that "strives to ensure diversity in the AES worldwide and the audio industry as a whole by improving accessibility, welcoming diverse genres, embracing emergent audio fields and research, and radiating inclusiveness to all races, genders and gender identities, physical abilities, ages, and nationalities." With the aim of providing this committee with detailed and global data to inform their future actions, our study elicits music producers', audio engineers', and studio assistants' experiences of social discrimination and microaggressions in the commercial recording studio.

We begin with a literature review that explores aspects of studio work that may contribute to or intensify in-

${ }^{1}$ https://www.gear-club.net/episodes/2019/tom-elmhirst

${ }^{2}$ http://www.aes.org/community/diversity/ 
equalities within the field, placing our survey and participants in context. Our methodological approach draws upon microaggression scholarship [8], [9] and intersectionality [10]-[14] to understand how gender, sexual orientation, race/ethnicity, migration, level of ability, and age impact studio professionals' work conditions and prospects of pursuing a successful career. Also, since our survey instrument mirrors one that was used for a study in Science, Technology, Engineering, and Mathematics (STEM) fields [1]-[3], we are able to compare the percentages of women producers, engineers, and assistants who face specific dimensions of gender-related microaggressions in the studio with those from STEM academia to underline the grim reality of our field.

\section{LITERATURE REVIEW}

\subsection{Emotional and Invisible Labor in the Studio}

Results from a 2008 online survey of an international cohort of young professional musicians (mean age $=26$, $\mathrm{n}=16$ ) showed a shared expectation that producers and engineers exhibit strong interpersonal and communication skills. These skills, which "allow trust and honesty in the studio" and "create a good atmosphere for performance," took precedence over technical, listening, and musical skills [15].

Similarly, based on semi-structured interviews with London-based producers and engineers (men, between the ages of 20 and $65, \mathrm{n}=19$ ), Watson and Ward [16] described the intimacy of recording studios as "emotional spaces characterized by trust and tolerance." They applied sociologist Hochschild's concept of emotional labor-the management of feelings and expressions based on the emotional requirements of jobs in the service industry [17] — to understand the job requirements of producers and engineers who, in the commercial recording studio, are "evokers of and witnesses to [musicians'] emotional displays that in most other work-based contexts, or even social contexts, would be considered inappropriate" [16]. To illustrate the scope of this emotional labor, producer Afanasieff [18] mentioned the need to be interchangeably like a doctor, spiritual adviser, psychologist, or bartender to help singers overcome their insecurity.

Jarrett's ethnography of jazz and country producers highlighted the position of the 'self-effacing producer' who "inhibits the emergence of 'the producer' as an animated body - a self or subjectivity who breathes life into sound" as opposed to the 'visible' producer, e.g., Phil Spector [19]. Findings from semi-structured interviews in Canada, France, and the USA with six renowned producers with more than 20 years of studio experience showed that they employ self-effacing methods to cope with musicians' sensitivities, including the possibility "to divert aggressiveness towards themselves" [20]. They compared their mission to the one of photographers "who aim to capture the most meaningful moments and then bring them together."

They also identified with "cleaners, servants, captains of a ship, firemen, and midwives," professions that engage in many forms of invisible labor ${ }^{3}$ and that are crucial to the functioning of the recording studio yet are elided or "invisible to those who are its beneficiaries." For instance, recording sessions require a great deal of preparation before the musicians arrive [21] and clean-up after the musicians leave (e.g., discarding coffee cups, rolling cables, backing up files). In short, music producers, audio engineers, and studio assistants engage in high levels of emotional and invisible labor that is underappreciated and unacknowledged.

\subsection{Intermediaries Between Musicians and the Industry}

In addition to developing a thick skin [22] and demonstrating resilience in their capacity to handle intimate and uncomfortable situations while performing emotional and invisible labor, studio professionals must follow the rules and conventions of the music industry in pursuing a studio career [23]. These rules and conventions are historically gendered [24], [25] and mirror the heteropatriarchal ${ }^{4}$ structure of this industry. Indeed, studio professionals act as "cultural intermediaries between production and consumption" [28], thus at the intersection of two male-dominated groups whose expectations and bias reinforce the gendering of the commercial recording studio, i.e., musicians across genres [29], [30] and music industry professionals [22].

Recent work emphasizes that the music industry's genius system maintains women and other historically and systemically marginalized groups of studio professionals in undermined and powerless positions [31], [32], to the point that Wolfe advocates for home-studio self-isolation as the healthiest solution for women producers to develop their skills and self-confidence before facing the gendered and heteropatriarchal commercial recording studio [22]. Furthermore the decline of global recording revenues since the early $21^{\text {st }}$ century has resulted in decreased budgets for recording projects, the need for producers to reinvent their profession [33], and the requirement to handle three jobs at once [34]. In this highly competitive industry, studio professionals are likely to be extremely protective of their clients, which further intensifies the exercise of exclusionary gendered power.

\subsection{The Power of Controlling Sound}

Whether sound recordists identify as 'tonmeisters' [35], 'traditionalists' who focus on capturing music performances, or 'technophiliacs' whose creative process is primarily technology driven [36], their work involves the control of sounds through audio technology. Horning emphasized the centrality and extent of tacit knowledge required to "engineer the performance" [38]. The power of controlling

\footnotetext{
${ }^{3}$ http://micemagazine.ca/issue-one/what-invisible-labour -mice-issue-01

${ }^{4}$ Patriarchy is "a system of social structures and practices in which men dominate, oppress, and exploit women" [26]. Heteropatriarchy is a social system ". . . in which heterosexuality and patriarchy are perceived as normal and natural, and in which other configurations are perceived as abnormal, aberrant, and abhorrent" [27].
} 
sound is thus connected with the legitimacy of coaching artists' performances. For instance, an ethnography of digital studios in Bamako (Mali) linked studio owners' access and ability to use technologies that are still rare and new in a low-income country and their legitimacy to coach artists and control the arrangement and composition process [39]. Wolfe [22] argues that the desire to retain control of the sound of the artist's voice and how that voice is presented is gendered and that "for the male producer to have held these particular creative reins for such a long time has also resulted in [...] an unwillingness to not only let go of them but also in a tendency to overlook and or dismiss the artist who takes control of them herself."

From his observations of three recording sessions for the creation of a pop song in a mid-sized studio, Gander spotlighted how the location of the producer's chair ('captain's chair') at the sonic sweet spot underlines the producer's control of space, restricting the musicians' involvement in the production process-judgments and decisions take place in the control room while the musicians are performing in the live room [40]. Minchella stated from a musician's perspective, "Space is so much more than the realm of surveyors and measurement; rather, it is central to our whole experience and is an intrinsic factor in the process of creativity itself" [41]. The link between the control of sound and control of space is somewhat reminiscent of Keightley's study of systemic sexism in Hi-Fi equipment ads in the mid-20 2 th century, e.g., "You are the Sultan with 70 Watts in your harem" [42]. Thus the space of the recording studio is intimately connected to issues of power, gender, and control.

\section{THEORETICAL FRAMEWORKS}

\subsection{Microaggression Theory}

The term microaggression was originally defined by Pierce, who was a professor of education and psychiatry at Harvard Medical School, to describe brief indignities that convey hostility toward a racialized group [43]. Importantly, micro refers to everyday rather than being lesser or insignificant [8]. Sue, professor of counseling psychology at Columbia, defined microaggressions as "everyday verbal, nonverbal, and environmental slights, snubs, or insults, whether intentional or unintentional, that communicate hostile, derogatory, or negative messages to target persons based solely upon their marginalized group membership" [9]. Sue and his team recently summarized the impacts of microaggressions on targets, ranging from increased stress, depression, and heavy toll on physical and emotional wellbeing to impeded learning and problem solving [44].

\subsection{Intersectionality}

Rooted in the writings of Black feminists and critical race scholars such as Crenshaw [10], Collins [11], [13], McCall [12], and Misra et al. [14], intersectionality is a theoretical approach, methodological orientation, and praxis. Rather than focus on a single form of social difference, intersectionality focuses on the 'matrix of domination' [11], and how systems of oppression are interlocking in nature. The guiding premises of intersectionality's "cognitive architecture" include: 1) Race, class, gender, sexuality, ability, and other systems of power are interdependent; 2) intersecting power relations produce complex social inequalities; 3 ) intersecting power relations shape group experiences; and 4) solving social problems requires intersectional analyses [13]. Specifically our project aims to document "the workings of power relations in producing social inequalities and the social problems they engender" [13] within the commercial recording studio by examining experiences of microaggressions through an intersectional lens.

\subsection{Research Questions}

RQ1. How do demographic categories such as gender (GEN), sexual orientation (SOR), race/ethnicity (RET), migrant status (MIG), gross national income of country of residence (GNI), disability (DIS), and the continuous demographic variable age (AGE) impact what tasks audio engineers accomplish within the studio (TAS), how well they are paid (DRA), and how often they are properly credited for their work (CRE)?

RQ2. How do demographic categories and variables impact music producers', audio engineers', and studio assistants' experiences of social discrimination and microaggressions in the commercial recording studio?

RQ3. How do women's experiences of microaggressions in the commercial recording studio compare with women's experiences of microaggressions in STEM academia?

RQ4. Within a framework of intersectionality, how do sexual orientation (SOR), race/ethnicity (RET), migrant status (MIG), gross national income of country of residence (GNI), disability (DIS), and age (AGE) intersect with gender (GEN) in impacting these experiences?

\section{METHODS}

\subsection{Survey Instrument Design}

Our survey design applies McCall's recommendations to use analytical categories strategically in order to document relationships of inequality among studio professionals ('intercategorical complexity') [12]. We constructed a survey instrument that includes a demographic portion and microaggressions portion with the option to opt out of the survey once the demographic portion is complete. In the $d e$ mographic portion featuring 17 close-ended and 10 openended questions we asked our respondents to self-identify in terms of gender (GEN), sexuality (SOR), race/ethnicity (RET), and disability status (DIS) and to answer questions about their age (AGE), country of origin and country of residence (MIG), years on the job (YOB), remuneration (DRA), how they were properly credited for their work [CRE, on a five-point Likert scale (LS1) from Almost always to Almost never], whether they received awards, and 
which studio tasks they were involved in (TAS, e.g., Tracking; Mixing; Assisting, Production).

In the microaggressions portion featuring 53 closeended questions using a five-point Likert scale (LS2) from Strongly disagree to Strongly agree, we captured studio professionals' experiences of microaggressions in the workplace. These 53 items drew upon 3 distinct survey instruments. Yang and Carroll [3] provided us with a breakdown of how they constructed their survey instrument. We also adapted Lewis and Neville's [1] factors-Assumptions of Beauty and Sexual Objectification (SOB), Silenced and Marginalized (SAM), and Strong Black Woman Stereotype (STE)—and Nadal's [2] Workplace and School Microaggressions (MGEN, MSOR, MRET, MAGE, MCUL, MDIS).

We met with the AES Diversity \& Inclusion Committee at the AES Conventions in Milan in May 2018 and New York in Oct. 2018 to implement changes to the survey instrument based on members' feedback. These changes included the possibility for respondents to share specific examples of microaggressions and how their experience of discrimination may have evolved over time. A week before we launched the survey we sent it to our personal network of 100+ studio professionals and asked for feedback. Several changes were integrated, including the addition of two open-ended questions about having witnessed or caused microaggressions in the studio. The survey questions and recruitment material were translated from English into 19 languages. The survey was available on Qualtrics from October $1^{\text {st }}$, 2019 to January $30^{\text {th }}, 2020$.

\subsection{Respondent Recruitment}

Aiming to capture a current and recent snapshot of studio professionals' experiences of discrimination in the commercial recording studio, our target population was individuals who had worked as producers, engineers, or studio assistants on other people's music in the last ten years. The primary group we intended to reach was the 12,800 members of the AES (about 9,100 professionals and 3,700 students), the largest professional society devoted exclusively to audio engineering. However, taking into account the realities of AES membership and participation [6], we also administered the survey to other communities of audio practitioners who more or less overlap with AES but who were likely to include more women and non-binary and/or gender non-conforming people, e.g., the Women's Audio Mission (WAM), SoundGirls, Audio Girl Africa, and female:pressure.

Our recruitment strategy involved three main aspects: emails to personal contacts, in-person recruitment of participants, and online dissemination of the survey via formal and informal channels. In-person recruitment took place at the 2019 AES New York Convention with a group of volunteers from a variety of gender and racial/ethnicity groups and at a series of four events organized by AES student chapters promoting the survey in the UK, Germany, and the Netherlands. The survey was disseminated online via emails to AES chapters worldwide and an email on the
VDT (Verband Deutscher Tonmeister) listserv and was included in an AES newsletter. Finally posts were made on group social media pages, including Hey Audio Student and Tape Op Magazine. Our goal was to collect a sample of roughly 1,000 respondents from across the globe. We did not expect nor desire to obtain a representative sample of the demographics of audio engineering globally but rather to obtain adequate sample numbers in order to understand the experiences of members of the various demographic categories of interest.

\subsection{Demographic Categories and Discrimination}

We defined six demographic categories and one continuous demographic variable as the independent variables for statistical analysis. The six demographic categories and their corresponding codes were gender (GEN), sexual orientation (SOR), race/ethnicity (RET), disability status (DIS), migrant status (MIG), and gross national income of country of residence (GNI). Age (AGE) was the final continuous demographic variable.

We defined fourteen dependent variables geared toward understanding the experiences of studio professionals' discrimination. The first five dependent variables were tasks undertaken in the studio (TAS), daily rate when working in the studio (DRA), crediting (CRE), AES membership (AESM), and awareness of the AES Diversity \& Inclusion Committee (AESD). Then followed nine microaggression factors, namely Assumptions of Beauty and Sexual Objectification (SOB); Silenced and Marginalized (SAM); Stereotyped (STE); and Workplace Microaggressions around Gender (MGEN), Age (MAGE), Race/ethnicity (MRET), Culture (MCUL), Sexual Orientation (MSOR), and Disability (MDIS).

\subsection{A Mixed-Method Analysis Approach 3.4.1 Descriptive Statistics}

We adopted a grounded approach [45] in order to code respondents' self-definitions into appropriate strategic categories. Gender (GEN) was coded from respondents' sex assigned at birth and their current gender identity into three categories: trans/non-binary, cisgender woman, and cisgender man. Sexual orientation (SOR) was coded into heterosexual and non-heterosexual (including but not limited to lesbian, gay, bisexual, queer, pansexual, and asexual). Based on responses to two questions that asked participants to specify their race/ethnicity and self-identify whether they were a racial minority in their workplace, categories of race/ethnicity (RET) detailed whether the respondent was a racial minority at their place of work and included: no, yes (BIPOC—Black, Indigenous, People of Color), and yes (white). This was further broken down into two categories: no (either white, or BIPOC and not part of a racial/ethnic minority in their place of work) and yes (both BIPOC and part of a racial/ethnic minority in their place of work). Note that BIPOC participants who did not identify as a racial minority in their place of work would here be included in the 'no' category for RET. Migration status (MIG) was coded 
as 'no' if a respondent's country of residence was the same as their country of origin and 'yes' if they differed.

We used the World Bank's classification of the economies of the country of residence into four income groups (GNI): high, upper middle, lower middle, and low. Disability status (DIS) was coded using responses to two questions, i.e., asking participants whether they identify as someone with disabilities and to further self-identify if they selected 'yes' to the previous question. For the purposes of statistical analysis we coded respondents into three categories: no (no disability), yes (yes invisible), and yes (visible). Age (AGE) and years on the job (YOJ) were calculated by subtracting the year of birth and year they started working in the studio, respectively, from 2020.

There are ethical and methodological issues involved in this strategy of categorization and aggregation. For example, the practice of including in one group both participants who are non-binary and those who have a binary gender (man or woman) but are also transgender is inherently problematic [46], [47]. Although the participants who we included in the category of 'trans/non-binary' had a wide variety of individual gender identities and experiencestrans woman, trans man, demigendered, non-binary, genderqueer, genderfluid, non-binary woman, and so onaggregating allowed us to obtain statistically significant results.

The same issues apply to the other strategic categories we employed. As critical race theorists and feminist scholars have pointed out, "race and gender frequently function as proxies: variables that reduce the complexities of biosocial bodily experience to more quantifiable forms of data" [46]. As with any strategic coding, these categories should be understood as permeable containers for complex and nuanced data. Note that in our forthcoming qualitative paper we unpack these strategic categories and analyze how these individuals experience microaggressions and discrimination through an intersectional lens.

The survey contained three questions related to income that were combined in order to calculate an average daily rate (DRA) in USD for each participant. Individuals who free-lanced in music production reported their normal daily rate as a freelancer and participants on payroll reported their normal yearly income, which we converted to a daily income. We normalized these numbers by the total percentage of their income that these participants reported making from audio engineering as opposed to other types of work. In the case of those who were both freelancers and on payroll we took an average value. We converted the currency to USD based on the value of that currency on the closing date of the survey. Six outliers who reported a freelancing daily rate of over 2,000 USD/day were removed, since inspection of their responses indicated that they had misinterpreted the question to refer to either a monthly or yearly income. Finally for the statistical tests we discarded the income information from participants who reported making less than $50 \%$ of their income from audio engineering.

For the coding of the Audio Engineering Society membership (AESM) we used the responses to a multiplechoice question on AES membership. The three categories for this variable include: no (never), yes (current member), and yes (former member). Participants were then asked to indicate whether they were aware of the AES Diversity \& Inclusion Committee (AESD). Responses were coded based on the coding assigned to AESM and included: no (current AES member), no (non-AES member), no (former AES member), yes (current AES member), yes (non-AES member), yes (former AES member), and N/A for those who skipped the question or left it blank.

\subsubsection{Statistical Analyses}

One-way Kruskal-Wallis tests and ANOVAs were used to relate demographic categories to CRE, DRA, AESM, and AESD. A logistic regression was used to relate AGE to TAS and $\chi^{2}$ tests for independence and cross tabulations were used to relate demographic categories other than AGE to TAS. Effect sizes for these $\chi^{2}$ tests were estimated using Cramer's V.

For each of the nine microaggression factors we took the average of all subsidiary questions in order to obtain an aggregate score for that factor. For each combination of demographic category and microaggression factor aggregate score we subsequently ran both one-way Kruskal-Wallis tests and one-way ANOVAs, followed by a Dunn's multiple comparison post-hoc test. Type III (sigma-restricted) sum of squares error terms were used for all ANOVAs. Use of ANOVAs was justified by making the approximation that Likert-scale measurements can be treated as continuous [48]. In the cases where the Kruskal-Wallis tests disagreed with the ANOVA findings, we reported the findings from the more conservative non-parametric Kruskal-Wallis test. We estimated the effect size for each test using bootstrapped $\eta^{2}$ values, referring to Cohen's [49] criteria for boundaries on low, medium, and large effect sizes.

For the continuous variable AGE, we ran a linear regression against each of the aggregate scores. Effect size was estimated using $\mathrm{R}^{2}$ statistics, again referring to Cohen for boundaries on effect size categories [50].

While aggregate scores were used to understand the overall impact of demographic categories upon microaggression factors, we were also interested in which of the specific microaggression statements were highly associated with demographic categories (e.g., the microaggression 'I have been told I was too sassy and straightforward' with its implications of racial stereotyping). As such we ran a second round of Kruskal-Wallis tests and ANOVAs on the disaggregated microaggressions data.

\subsubsection{Comparison With STEM Study}

To compare our findings with those obtained by Yang and Carroll [3], who measured the experiences of women working within STEM academia, we reproduced their calculations. However, while our survey tool employed a 5-point Likert scale ranging from Strongly disagree to Strongly agree, theirs employed a 7-point scale on the same range, with their score of 4 (Neither agree nor disagree) corresponding to our score of 3 (Neither agree nor disagree). 
Table 1. Demographic breakdown of strategic categories

\begin{tabular}{|c|c|c|c|c|}
\hline $\begin{array}{l}\text { Demographic } \\
\text { Categories }\end{array}$ & $\begin{array}{l}\text { Cisgender } \\
\text { Man }\end{array}$ & $\begin{array}{l}\text { Cisgender } \\
\text { Woman }\end{array}$ & $\begin{array}{c}\text { Trans/Non } \\
\text { Binary }\end{array}$ & Total \\
\hline Total & 275 & 86 & 26 & 387 \\
\hline Low GNI & 10 & 0 & 2 & 12 \\
\hline Lower Mid GNI & 9 & 4 & 0 & 13 \\
\hline Upper Mid GNI & 42 & 14 & 2 & 58 \\
\hline High GNI & 214 & 68 & 22 & 304 \\
\hline Immigrant & 46 & 12 & 5 & 63 \\
\hline $\begin{array}{l}\text { Both BIPOC \& part of } \\
\text { racial/ethnic minority } \\
\text { in place of work }\end{array}$ & 18 & 11 & 2 & 31 \\
\hline Non-heterosexual & 47 & 44 & 19 & 110 \\
\hline Person with disabilities & 15 & 12 & 5 & 32 \\
\hline AES member & 108 & 30 & 6 & 144 \\
\hline $\begin{array}{c}\text { Aware of AES D\&I } \\
\text { Committee }\end{array}$ & 61 & 14 & 7 & 82 \\
\hline
\end{tabular}

\subsubsection{Intersectional Analyses}

To explore how the demographic categories intersect with gender in experiences of microaggressions in the studio, we ran two-way ANOVAs with Type III sum of squares error to test for the following interactions: GEN/SOR, GEN/RET, GEN/DIS, GEN/MIG, and GEN/GNI. To investigate the intersection between GEN/AGE, we ran an ANCOVA with hierarchical error terms.

\section{RESULTS}

\subsection{Descriptive Statistics}

A total of 1,141 participants started the survey. We analyzed only the data from participants who completed the demographic portion (GEN, SOR, RET, DIS, MIG, GNI, AGE, TAS, CRE, DRA, AESM, and AESD) of the survey in its entirety, resulting in 387 usable responses, 373 of which included usable age data (Table 1). Out of these 387 participants, 281 completed the first microaggressions portion (SOB, SAM, and STE) and 234 completed the second microaggressions portion (MGEN, MSOR, MRET, MDIS, MCUL, and MAGE). Despite not reaching our goal in terms of participation, proportionally more participants from underrepresented populations than are present in the profession at large allowed us to obtain meaningful results from the data nevertheless.

\subsubsection{GEN, SOR, RET, DIS, MIG, GNI, AGE}

Out of the 387 participants who filled out the demographic portion, $71 \%(\mathrm{n}=275)$ of participants were cisgender men, $22 \%(\mathrm{n}=86)$ were cisgender women, and $7 \%(\mathrm{n}$ $=26$ ) were transgender/non-binary. We received responses from approximately $15 \%$ more cisgender women than have been estimated to comprise the overall gender makeup of AES [4]. Also, 17\% $(n=47)$ of cisgender men, $44 \%(n$ $=38)$ of cisgender women, and $73 \%(\mathrm{n}=7)$ of trans/nonbinary participants identified as non-heterosexual.

Out of the 387 participants, $8 \%(n=31)$ were both BIPOC and part of a racial/ethnic minority in their place of work- $45 \%$ of whom had migrated from their country of birth-while $89 \%(\mathrm{n}=344)$ did not identify as part of a racial/ethnic minority in their place of work. Finally

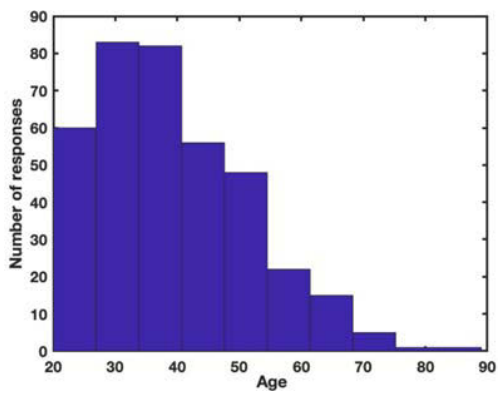

Fig. 1. AGE demographics of survey respondents, $n=373$.

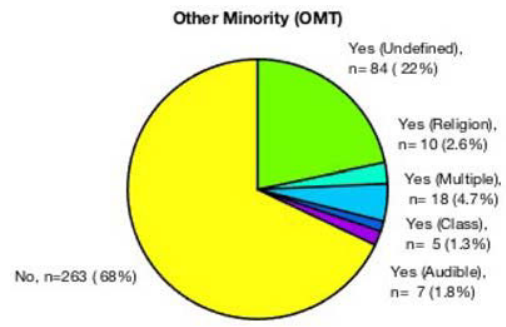

Fig. 2. Other minority statuses reported by study participants not included in the analysis undertaken in this study.

$3 \%(\mathrm{n}=12)$ were white but also identified as a racial minority in their place of work. In the statistical analysis that follows, this cohort was combined with the group that did not identify as a racial minority in their workplace.

Our sample was international, comprising data from participants born in 36 countries of origin and residing in 46 countries of residence, $94 \%$ from upper middle and highincome GNI countries (Table 1). Out of the 387 participants, $16 \%(n=63)$ reported living in a country different from the one they were born in. Of these, $22 \%$ identified as both BIPOC and part of a racial/ethnic minority in their place of work. Three times as many cisgender men from low and low-middle income GNI countries participated in the survey, as did cisgender women or transgender/non-binary people ( $2 \%$ difference).

Out of the 387 participants, $8 \%(n=32)$ identified as having a disability. Of these individuals just over half reported having a visible physical disability while the rest reported having an invisible physical or non-physical disability.

The mean age that the participants started working in audio engineering was $25[ \pm 5]$ years old. There was no significant difference in this value with respect to gender. The youngest participant was 20 years of age and the oldest was 89 years of age (Fig. 1).

Participants were given a space to indicate whether they identified as part of a minority in their place of work other than the demographic categories included in the survey (Fig. 2). We did not include the demographic categories featured in the responses to this question in our statistical analysis because either the number of respondents was very small (for audible minority, religious minority, and class minority) or because while the participants answered that they were part of another minority group they did not specify what kind. Additionally, while we included a question 
about Indigenous status in the survey, we did not include the results in our statistical analysis because this question was misinterpreted (sometimes in ways that seemed intentionally provocative or anti-Indigenous) by a large number of participants.

\subsection{Tasks, Incomes, and Credits}

\subsubsection{Tasks (TAS)}

Statistically significant differences in the distribution of tasks accomplished in the studio (TAS) were found for gender (GEN), sexual orientation (SOR), disability (DIS), and AGE (Table A1). Overall, cisgender women were $25 \%$ more likely to answer assisting than cisgender men; however when we included only participants under the age of 30 in the analysis, we found the result to not be statistically significant, indicating that this finding was mainly due to the small number of older women who participated in the survey. Cisgender men were $21 \%$ more likely to answer mastering than cisgender women; this result was independent of age ( $p$ $<\mathrm{e}-2)$.

Heterosexual participants were $17 \%$ more likely to answer mastering $(p<\mathrm{e}-2), 11 \%$ more likely to answer producing $(p=0.05), 9 \%$ more likely to answer mixing ( $p<$ $\mathrm{e}-2)$, and $17 \%$ less likely to answer assisting $(p<\mathrm{e}-2)$ than non-heterosexual participants; these results were independent of age.

Participants who did not identify as having a disability were more likely to respond mixing than participants with an invisible or visible disability $(19 \%$ and $16 \%$, respectively, with $p=0.01$ ).

Age had a significant impact on how often participants responded mixing (increased with age, $p<\mathrm{e}-8$ ), mastering (increased with age, $p<0.05$ ), and assisting (decreased with age, $p<\mathrm{e}-18)$.

\subsubsection{Incomes (DRA)}

Both gender and age were significantly associated with differences in daily rate $\left(\mathrm{GEN}, \eta^{2}=0.07\right.$ [0.01:0.10], medium effect size; AGE, $\mathrm{R}^{2}=0.14$, medium effect size). Cisgender men making over $50 \%$ of their income from audio made an average of $74 \pm 4$ USD/day, while trans/nonbinary people made an average of $56 \pm 13 \mathrm{USD} /$ day and cisgender women made an average of $51 \pm 7$ USD/day. Daily rate increased with age at a rate of $3.74 \mathrm{USD} /$ day per year (Fig. 3, Table 2).

\subsubsection{Crediting (CRE)}

Gender, race/ethnicity, disability, migrant status, and age were found to be significant predictors of being properly credited for work (CRE) (Fig. 4). Cisgender women were twice as likely as cisgender men to report being almost never properly credited and half as likely to report being almost always properly credited (Tables 2 and A2; $\eta^{2}$ $=0.06$ [0.02:0.12], medium effect size). Participants who were both BIPOC and part of a racial/ethnic minority in their place of work were just under twice as likely as participants who were not to report almost never being properly credited and under half as likely to report being almost always properly credited $\left(\eta^{2}=0.02\right.$ [0.002:0.06], small effect size).

Participants with an invisible disability were almost four times more likely than participants without a disability to respond almost never and one-fifth as likely to respond $a l$ most always being properly credited for their work $\left(\eta^{2}=\right.$ 0.02 [0.005:0.07], small effect size). Participants who had emigrated were $10 \%$ more likely to respond almost never or rarely and $10 \%$ less likely to respond almost always being properly credited than those who had not emigrated $\left(\eta^{2}=\right.$ 0.02 [0.001:0.06], small effect size). Older participants reported being properly credited significantly more often than younger participants $\left(\mathrm{R}^{2}=0.02\right.$, small effect size $)$. Additionally two-way analysis of GEN/AGE vs. CRE revealed that there was a significant interaction term for GEN*AGE. While the overall effect of increasing age was to positively affect how often participants were properly credited, the intersection of gender and age had a small additional mediating effect - in the case of cisgender participants this effect was negative, while for trans/non-binary participants it was positive.

\subsection{AES Membership}

The only significant predictor of AES membership (AESM) was GNI of country of residence (Tables 2 and A2). Participants from low and lower-middle income GNI countries were significantly less likely to be AES members than participants from upper-middle and high-income GNI countries (Table A2). Only 55\% of current AES members who took part in the survey were aware of the AES D\&I committee. None of the demographic categories we tested were significant predictors of knowledge of the AES D\&I committee.

Of the participants who indicated that they had never been a member of AES, about $33 \%$ provided a reason why. Reasons included already being members of the VDT, considering becoming members in the future, not being interested in joining AES, not being able to afford a membership, stating that the membership was not worth the return on investment, participating in AES but not as a member, not being able to register for a membership on the AES website, and not knowing what AES is. For participants who were formerly members of AES but no longer active, about $85 \%$ explained why. Some cited fiscal reasons for not continuing their membership, such as not being able to afford dues, not having a return on investment, and not being interested in the benefits. Several respondents indicated that they had an AES student membership during their studies but did not renew it after their graduation.

Other reasons given for not having a current membership included letting their membership lapse/forgetting to renew, not being a part of the audio industry anymore, and having a grievance with the society (i.e., not feeling supported, issues with exclusion, and too oriented toward technical or social aspects). Lastly eight respondents included comments on their answer to their awareness of the Diversity \& Inclusion 

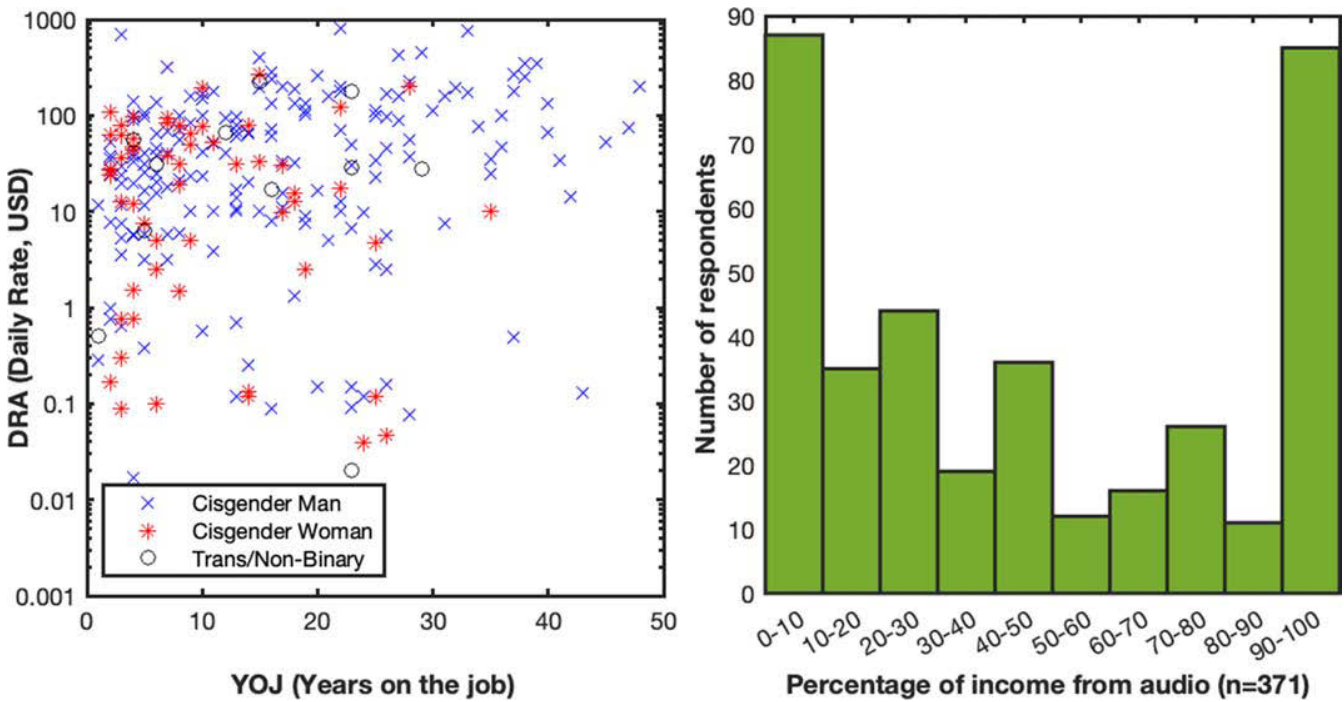

Fig. 3. Left: daily rate (DRA, USD/day) vs. years on the job. Right: percentage of their income that participants reported making from audio engineering.
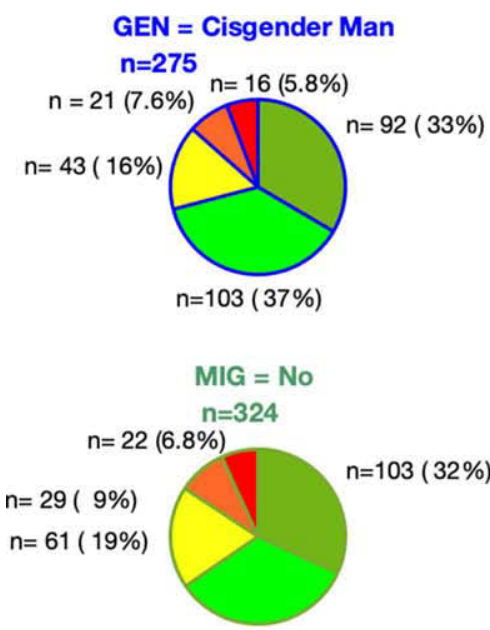

$n=109(34 \%)$
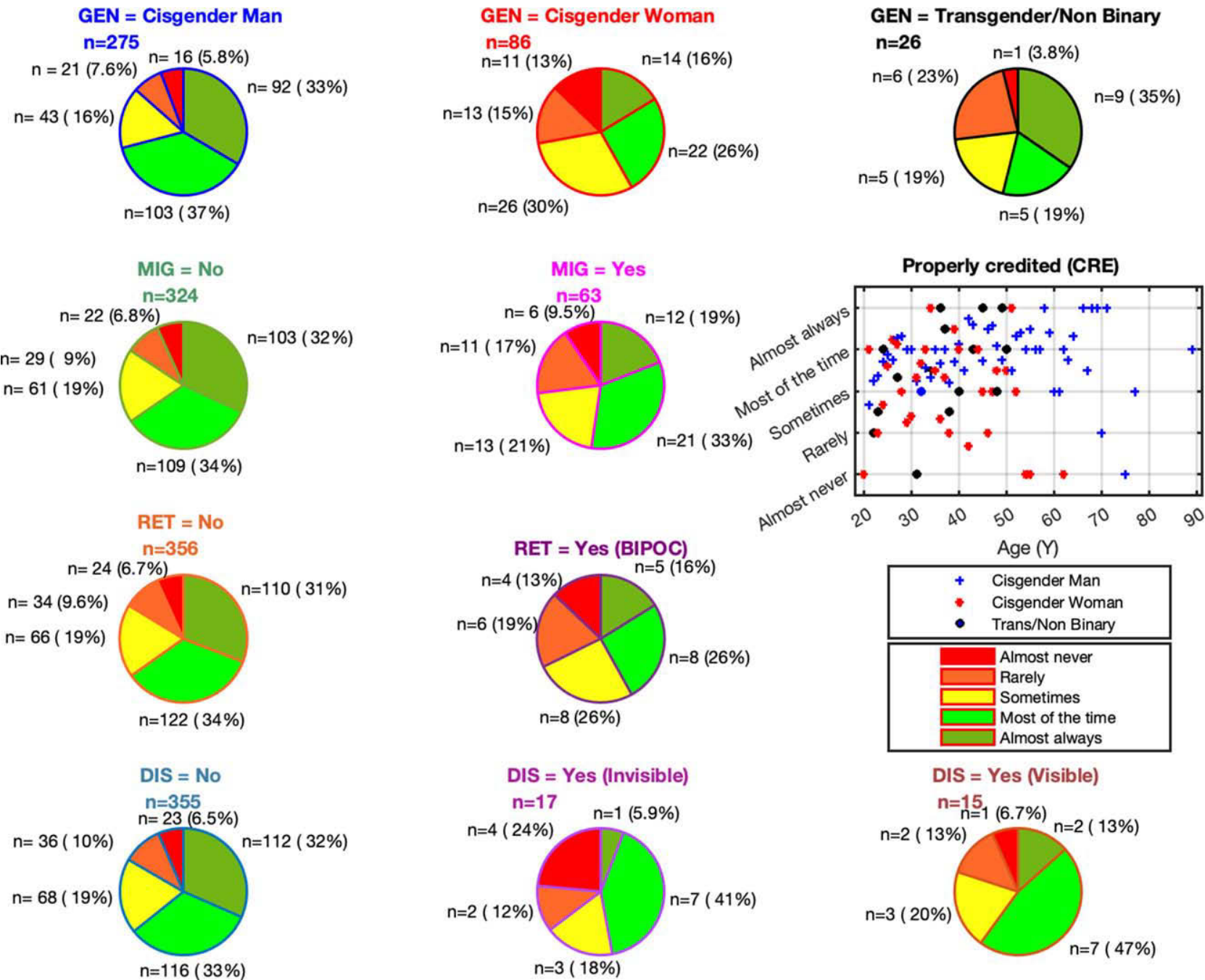

Fig. 4. Demographic factors associated with difference in CRE. For AGE subplot, markers represent the mean response for a given gender at a given age. 
Table 2. P-values for $\chi^{2}$ values (one-way Kruskal-Wallis tests; GEN/SOR/RET/DIS/MIG/GNI) and $\mathrm{R}^{2}$ values (linear regressions; AGE), color coded by effect size. Entries with background in dark gray/red indicate large effect size $\left(\eta^{2}>=0.1379\right.$ or $\left.R^{2}>0.26\right)$, entries in medium gray/orange indicate medium effect size $\left(\eta^{2}>=0.0588\right.$ or $\left.\mathrm{R}^{2}>0.13\right)$, entries in light gray/yellow indicate small effect size $\left(\eta^{2}>=0.0099\right.$ or $\left.R^{2}>0.02\right)$. Non-significant entries are in white with gray text. Slope entry color indicates direction of relationship of dependent variable with AGE (light gray/pink positive, dark gray/blue negative)

\begin{tabular}{|c|c|c|c|c|c|c|c|c|c|c|}
\hline & & \multirow[t]{2}{*}{$\mathbf{N}$} & GEN & SOR & RET & DIS & MIG & GNI & \multicolumn{2}{|c|}{ AGE } \\
\hline & & & \multicolumn{6}{|c|}{$\mathbf{p}\left(\chi^{2}\right)$} & $\mathbf{p}\left(\mathbf{R}^{2}\right)$ & Slope \\
\hline Daily rate & DRA & 135 & 0.01 & 0.96 & 0.09 & 0.47 & 0.43 & 0.29 & $5.86 \mathrm{E}-0.6$ & 3.74 \\
\hline Properly credited & CRE & \multirow{3}{*}{373} & $1.05 \mathrm{E}-03$ & 0.11 & 0.005 & 0.01 & 0.01 & 0.64 & 0.01 & 0.01 \\
\hline AES membership & AESM & & 0.27 & 0.43 & 0.31 & 0.24 & 0,29 & 0.05 & 0.49 & $2.19 \mathrm{E}-3$ \\
\hline Aware of D\&I committee & AESD & & 0.43 & 0.34 & 0.92 & 0.39 & 0.26 & 0.15 & 0.50 & $4.46 \mathrm{E}-3$ \\
\hline Sexual objectification & SOB & 283 & $1.20 \mathrm{E}-16$ & $5.05 \mathrm{E}-04$ & 0.14 & $1.68 \mathrm{E}-03$ & 1.00 & 0.34 & 0.01 & -0.01 \\
\hline Silenced and marginalized & SAM & 284 & $1.26 \mathrm{E}-13$ & 0.03 & 0.03 & 0.01 & 0.44 & 0.20 & $8.68 \mathrm{E}-05$ & -0.02 \\
\hline Stereotyped & STE & 281 & $4.96 \mathrm{E}-11$ & 0.15 & 0.56 & 0.02 & 0.81 & 0.03 & 0.14 & -0.01 \\
\hline Gender W.M. & MGEN & \multirow{6}{*}{234} & $3.15 \mathrm{E}-25$ & $3.15 \mathrm{E}-05$ & 0.01 & 0.02 & 1.00 & 0.99 & $4.26 \mathrm{E}-03$ & -0.02 \\
\hline Age W.M. & MAGE & & $2.28 E 07$ & 0.04 & 0.64 & 0.08 & 0.96 & 0.24 & 4.73E-04 & -0.03 \\
\hline Sexual orientation W.M. & MSOR & & $1.06 \mathrm{E}-06$ & $2.99 \mathrm{E}-08$ & 0.10 & 0.00 & 0.66 & 0.50 & 0.11 & -0.01 \\
\hline Cultural W.M. & MCUL & & 0.02 & 0.73 & 0.09 & 0.01 & 0.01 & 0.02 & 0.97 & $-2.10 \mathrm{E}-4$ \\
\hline Disability W.M. & MDIS & & $4.35 \mathrm{E}-04$ & 0.07 & 0.34 & $2.26 \mathrm{E}-04$ & 0.78 & 0.68 & 0.01 & -0.02 \\
\hline Racial W.M. & MRET & & 0.05 & 0.52 & 0.01 & 0.04 & 0.51 & 0.01 & 0.42 & $-3.99 \mathrm{E}-3$ \\
\hline
\end{tabular}

committee (three who answered 'no' and five who answered 'yes'). Of the 'no' responses, reasons included not being aware of the committee, not being aware of the society, and not supporting the committee's endeavors. Of the 'yes' responses, two were from active committee members, two wrote in support of the committee, and one acknowledged its existence.

\subsection{Microaggression Statistical Analyses}

\subsubsection{Gender (GEN)}

Of the demographic categories considered in this analysis we found that gender was by far the strongest predictor of experiences of discrimination and microaggressions in the recording studio (Table 2), having large and medium effects upon aggregate scores for all microaggression factors except Race/Ethnicity Workplace Microaggressions (MRET). Four microaggression factors displayed large effect sizes for GEN: Assumptions of Beauty and Sexual Objectification (SOB, $\eta^{2}=0.25$ [0.19:0.38]), Silenced and Marginalized (SAM, $\eta^{2}=0.21[0.13: 0.30]$ ), Stereotyped (STE, $\eta^{2}$ $=0.17[0.10: 0.26])$, and Gender Workplace Microaggressions (MGEN, $\eta^{2}=0.48$ [0.44:0.67]), corresponding to an explained variance of $25 \%, 21 \%, 17 \%$, and $48 \%$, respectively.

For microaggression factors SOB, SAM, STE, MGEN, MAGE, and MSOR, the aggregate scores for cisgender women and trans/non-binary participants were significantly higher than those of cisgender men but not significantly different from one another. However trans/non-binary participants responded similarly to cisgender men for MDIS while cisgender women reported experiencing significantly more microaggressions from this factor. The opposite was true for MCUL, where cisgender women and cisgender men did not respond significantly differently from one another but where trans/non-binary participants reported higher incidences (Table A2).

\subsubsection{Sexual Orientation (SOR)}

Non-heterosexual participants reported experiencing significantly more microaggressions from factors SOB, SAM, MGEN, MAGE, and MSOR than heterosexual participants (Tables 2 and A2). The most influenced factors associated with SOR were MSOR $\left(\eta^{2}=0.13\right.$ [0.07:0.28], medium/large effect size $)$ and MGEN $\left(\eta^{2}=\right.$ 0.07 [0.03:0.18], medium effect size).

\subsubsection{Race and Ethnicity (RET)}

Participants who were both BIPOC and part of a racial/ethnic minority in their place of work reported experiencing significantly more microaggressions from factors SAM, MGEN, and MRET than participants who were not (Tables 2 and A2). The most influenced factors associated with RET were MGEN $\left(\eta^{2}=0.03\right.$ [0.0003:0.06], small effect size $)$ and MRET $\left(\eta^{2}=0.03\right.$ [0.007:0.18], small effect size).

\subsubsection{Migrant Status (MIG)}

Participants who had migrated were significantly more likely to experience Culture Workplace Microaggressions (MCUL) than participants who had not (Tables 2 and A2; $\eta^{2}=0.03$ [0.003:0.13], small effect size).

\subsubsection{Gross National Income of Country of Residence (GNI)}

Participants living in low and lower-middle income countries experienced significantly more microaggressions for factors STE, MCUL, and MRET than those living in upper middle and high-income countries (Tables 2 and A2). The most influenced factor associated with GNI was MRET $\left(\eta^{2}\right.$ $=0.03[0.001: 0.11]$, small effect size).

\subsubsection{Disability (DIS)}

Participants with an invisible disability were significantly more likely to experience microaggressions from 
Table 3. Comparison with Yang and Carroll's study [3] of experiences of microaggressions in STEM academia.

\begin{tabular}{|c|c|c|c|c|}
\hline \multicolumn{5}{|c|}{$\begin{array}{l}\text { Percentage of women respondents whose answers within } \\
\text { a microaggression factor resulted in an average } \\
\text { of 'Neither agree or disagree' or over. }\end{array}$} \\
\hline Study & SOB & SAM & & MGEN \\
\hline Yang \& Carroll & $31 \%$ & $47 \%$ & & $44 \%$ \\
\hline Current & $55 \%$ & $80 \%$ & & $77 \%$ \\
\hline \% Difference & $+24 \%$ & $+33 \%$ & & $+33 \%$ \\
\hline \multicolumn{5}{|c|}{$\begin{array}{l}\text { Percentage of women respondents who answered at least } \\
\text { once 'Agree' or above within a microaggression factor }\end{array}$} \\
\hline Study & SOB & SAM & STE & MGEN \\
\hline Yang \& Carroll & $73 \%$ & $76 \%$ & $76 \%$ & $68 \%$ \\
\hline Current & $82 \%$ & $93 \%$ & $87 \%$ & $82 \%$ \\
\hline$\%$ Difference & $+9 \%$ & $+17 \%$ & $+11 \%$ & $+14 \%$ \\
\hline
\end{tabular}

factors SOB, SAM, STE, MGEN, MSOR, MCUL, and MRET than participants with no disability (Tables 2 and A2). Additionally participants with either visible or invisible disabilities were significantly more likely to experience Disability Workplace Microaggressions (MDIS, $\eta^{2}=0.07$ [0.04:0.16], medium effect size).

\subsubsection{Age (AGE)}

Age had a significant negative association with experiences of microaggressions from factors SOB, SAM, MGEN, MAGE, and MDIS (Table 2). For these factors, increasing age was associated with a decrease in reported experiences of microaggressions. The most influenced factors associated with AGE were SAM $\left(\mathrm{R}^{2}=0.05\right.$, small effect size) and MAGE $\left(\mathrm{R}^{2}=0.05\right.$, small effect size $)$.

\subsection{Comparison With STEM}

We compared our findings with those reported by Yang and Carroll's study [3] and found that cisgender women working in the studio reported experiencing more microaggressions and discrimination than cisgender women working in STEM academia (Table 3). Specifically, 24\%, 33\%, and $33 \%$ more cisgender women working in the studio responded with an average of Neither disagree nor agree or over for microaggression factors SOB, SAM, and MGEN, respectively. Also, 9\%, 17\%, 11\%, and 14\% more of the cisgender women we surveyed had experienced one or more microaggressions from factors SOB, SAM, STE, and MGEN, respectively.

\subsection{Intersection Analyses}

We found significant simultaneous effects and interactions with gender for all demographic categories: GEN/SOR (Fig. A1), GEN/RET (Fig. A2), GEN/MIG (Fig. A3), and GEN/GNI (Fig. A4), GEN/DIS (Fig. A5), and GEN/AGE (Fig. A6). Microaggressions with simultaneous effects (figure titles in black) were unidirectionally impacted by both demographic categories. For example cisgender women and trans/non-binary participants reported experiencing the microaggression 'People have imitated the way that I speak' more than cisgender men, and non- heterosexual participants experienced this microaggression less than heterosexual participants (Fig. A1).

Microaggressions with an interaction term (purple and orange figure titles) showed more complex relationships to the demographic variables, e.g., 'I have been disrespected,' for which there was a significant term for GEN (cisgender men were the least likely to experience this microaggression) but none for SOR. However non-heterosexual cisgender women and trans/non-binary people reported experiencing this microaggression more than their heterosexual counterparts, whereas non-heterosexual cisgender men reported experiencing the microaggression less than heterosexual cisgender men-a dynamic captured by the GEN*SOR interaction term.

\subsubsection{Intersection GEN/SOR}

In the majority of cases where there was a simultaneous effect of GEN and SOR, non-heterosexual participants experienced the microaggression in question more than their heterosexual peers, and this difference was bigger for cisgender women and trans/non-binary people than cisgender men. However for the microaggressions 'People have imitated the way that I speak' and 'I have been told I was too sassy and straightforward,' non-heterosexual participants experienced these microaggressions less than heterosexual participants. Non-heterosexual cisgender women and trans/non-binary people experienced the SAM microaggressions 'I have been disrespected' and 'People have challenged my authority' more than their heterosexual peers whereas the opposite was true for cisgender men.

Finally, for the SOB microaggression 'People have made sexually inappropriate comments,' non-heterosexual cisgender participants experienced this microaggression less than heterosexual cisgender participants, whereas nonheterosexual trans/non-binary participants experienced it more than heterosexual trans/non-binary participants. $P$ values and effect sizes for the two-way ANOVA results for GEN/SOR can be found in Table A3, APPENDIX A.

\subsubsection{Intersections GEN/RET, GEN/MIG, GEN/GNI}

Participants who were both BIPOC and part of a racial/ethnic minority in their place of work experienced the MCUL microaggression 'People have treated me differently from other cultural groups' more often than those who were not. Cisgender men and trans/non-binary participants who were both BIPOC and part of a racial/ethnic minority in their place of work experienced the STE microaggression 'I have been told I was too independent' more than their peers who were not, while the opposite was true for cisgender women.

Participants who had migrated were more likely than those who had not to experience the STE microaggression 'People have made me feel exotic' but less likely to experience the SOB microaggression 'People have assumed I was sexually promiscuous.' They were also more likely to experience the MCUL microaggressions 'People have assumed my work would be inferior to people of other cultural origins,' 'People have been unfriendly or unwel- 
coming toward me because of my cultural origin,' and 'My opinion has been overlooked in a group discussion because of my cultural origin'; this effect was greater for cisgender women than cisgender men or trans/non-binary people.

Participants from low and lower-middle GNI countries were more likely than those from upper-middle and high GNI countries to have experienced the microaggressions 'People have imitated the way that I speak,' 'People have treated me differently than people of other cultural groups,' and 'People have treated me differently than people of other racial groups,' and less likely to have experienced the microaggressions 'People have made sexually inappropriate comments' regardless of gender. Cisgender participants from low and lower-middle GNI countries were more likely than those from upper-middle and high GNI countries to have experienced the microaggressions 'I have been disrespected,' 'People have challenged my authority,' 'I have been ignored because of my cultural origin,' 'I have been ignored because of my race,' and 'People have been unfriendly or unwelcoming towards me because of my level of ability,' whereas trans/non-binary participants from low and lower-middle GNI countries were less likely to have experienced these microaggressions.

Finally cisgender women from low and lower-middle GNI countries were more likely than those from uppermiddle and high GNI countries to have experienced the microaggressions 'My opinion has been overlooked in a group discussion because of my sexual orientation' and 'People have assumed my work would be inferior to people of other levels of ability,' whereas the opposite was true for cisgender men and trans/non-binary participants. Note that there were only two trans/non-binary respondents from lower GNI countries, so further investigation is required to generalize these results for transgender and non-binary people.

\subsubsection{Intersection GEN/DIS}

Participants with visible or invisible disabilities experienced the microaggressions 'People have objectified me based on my physical features,' 'I have received unwanted comments about my physical appearance,' 'I have received unwanted comments about my hair and/or makeup,' 'People have tried to "put me in my place," and 'I have been told I was too emotional' more than participants without disabilities. Participants with invisible disabilities were more likely than those with visible disabilities to report the first three of these microaggressions while the opposite was true for the last two.

Cisgender men with invisible disabilities were more likely than any other group to report the microaggression 'People have made me feel exotic.' Along with trans/nonbinary people with invisible disabilities, they were also more likely to report 'People have been unfriendly or unwelcoming towards me because of my cultural origin' or 'People have been unfriendly or unwelcoming towards me because of my race'-but the very low number of invisibly disabled trans/non-binary respondents to these questions $(n=1$ for the first and $n=2$ for the sec- ond) means it is difficult to generalize these results for the trans/non-binary case.'

\subsubsection{Intersection GEN/AGE}

Seven microaggressions showed simultaneous effects for both GEN and AGE, four from factor SAM and three from factor Age Workplace Microaggressions (MAGE). Younger cisgender women and trans/non-binary people reported experiencing these microaggressions more than older cisgender men. Older cisgender women also reported experiencing the microaggressions 'People have made me feel exotic' and 'My professional expertise has been questioned,' whereas middle-aged participants of all genders reported experiencing the microaggression 'People have been unfriendly or unwelcoming towards me because of my cultural origin' while younger and older participants did not.

\section{DISCUSSION}

\subsection{Limitations}

In this survey, we strategically used intercategorical complexity [12] to document social inequalities between different social groups within the commercial recording studio. From our perspective we use quantitative methods as a tool to advocate for change within the audio industry. This is akin to gear or technology in the studio. Although there are economic, social, and historical reasons that have resulted in gear and technology being racialized and gendered in particular ways, similar to methods, this does not exclude marginalized groups from using these tools in productive ways [14].

The biggest limitation of our survey is the low number of BIPOC participants. As an all-white research team of non-binary people and cisgender women, it is important to seriously examine this limitation of our dataset. First, it is possible to argue that the racial and ethnic make-up of our survey merely reflects the white supremacist heteropatriarchal nature of the audio industry. However we know the industry is also male-dominated and this did not stop women and non-binary people from participating.

Second, perhaps we can say that the underrepresentation of BIPOC is the result of recruitment issues. Several BIPOC AES student members assisted with our recruitment efforts (both in-person at the 2019 NY AES Convention and virtually through their social networks). However we know that recruitment practices can reflect systemic inequities. For example in medical research a study showed that Black respondents are less trusting of investigators than white respondents [51]. Similar issues of distrust may have been at play in our recruitment process.

Third, perhaps it is the nature of the methods themselves. We know that many theories, structures, and methods in academia show the bias of racism and white supremacy [52] and are also colonialist in nature [53], [54]. We suggest that BIPOC may have purposefully chosen not to participate in our survey as a result of this history and due to ongoing racist and colonial legacies within the academy. In conclu- 
sion, further studies investigating how BIPOC experience microaggressions in the recording studio are needed.

\section{2 'Under Pressure': Emotional and Invisible Labor}

Our findings point to an apparent contradiction between studio professionals' ability to regulate their own emotions in order to provide the trust and tolerance necessary to help musicians perform at their best [15], [16], [18]-[20], and the lack of trust and tolerance that trans/non-binary people, cisgender women, younger people, BIPOC, people with disabilities, and sexual minorities face when working in the studio. The adverse effects of emotional labor such as displaying accepted rather than real emotions may explain this contradiction. Indeed, the accompanying experience of emotional dissonance is associated with negative outcomes within the workplace, including emotional exhaustion, decreased organizational attachment, and burnout [55], all of which are predictors of workplace incivility [56]-[59].

While our findings show that women and other equitydeserving groups are less credited and compensated for their studio contributions, we also found that just under $15 \%$ of cisgender men reported being Almost never or Rarely credited for their work, and regardless of demographic category, the majority of studio professionals made an income of well under $100 \mathrm{USD} /$ day, with an increase of about 40 USD/day of their general revenues every ten years. Perceived job insecurity has been shown to be a motivating factor for workplace incivility [59], and experiencing incidences of workplace incivility is a predictor of instigating workplace incivility oneself [57]. This suggests that some studio professionals who silence, belittle, harass, or insult their peers may be responding to their own frustration and insecurities related to the precarious and invisible nature of their labor.

\subsection{Dangers of Heteropatriarchy in the Studio}

Following the highly significant impact of gender, our analysis indicates that age is the second most important factor influencing experiences of discrimination and microaggressions in the commercial recording studio. This mirrors the heteropatriarchal structure of the studio that requires young professionals to develop a 'thick skin' [22] and embrace the rules and conventions of the workplace to pursue a career in this informal industry [23]. While masculinity is not intrinsically harmful and can be associated with positive and prosocial traits such as helpfulness, courage, and responsibility [60], some typically masculine workplace behaviors such as competitiveness, assertiveness, and ruthlessness can become toxic when they are performed in "socially destructive" and dominating ways [61]. In male-dominated and competitive fields, toxic masculinity presents itself through extreme behaviors of competition and control, often an attempt to "prove manhood" at work [61], [62].

One of the outcomes of male-dominated toxic workplace cultures are 'queen bee behaviors,' which are "a response to the discrimination and social identity threat that women may experience in male-dominated organizations" [63]. This phenomenon leads women "to achieve career success in the field by derogating other women while simultaneously emphasizing their own career commitment and masculine qualities" [64]. This may explain the finding from a recent study exploring the factors contributing to gender imbalance in the audio industry that shows how women employees were less likely than men employees to believe that women would be suitable for technical and managerial roles [65].

This may also provide insight toward interpreting a result from another gender bias study that explored the experience of women in the music industry, which found that the $7 \%$ of the surveyed women who listed their occupation as 'music production and recording' reported a higher level of job satisfaction than average [25], [66]. These observations suggest that structural changes are needed to avoid the 'thick-skinned resignation' [22] that results from the 'queen bee' [63], [64] and 'tokenism' [67] phenomena.

\subsection{Toxic Masculinity as Gate Threshold}

Together our statistical results demonstrate how harsh and toxic the climate of the commercial recording studio can be for women, trans/non-binary people, BIPOC, immigrants, disabled people, non-heterosexual people, and younger people working in the field. As previously noted the association between masculinity and audio technology has insidious implications ranging from $\mathrm{Hi}-\mathrm{Fi}$ addictions that repulse spouses within the home [42] to the mobilization of gear fetishism that excludes women from the workplace. 'Audiomania' may also be accompanied by a certain risk aversion for new technologies [36] and is reflected in 'informal demarcations among audio engineers,' such as knowing how to roll cables over/under or being able to hear autotune artifacts [68]. Our findings contradict and challenge the 'genderless' central mission of audio engineering and music production, which has been conceived of as the use of technology in the service of art [69], i.e., to 'catch a vibe' [22] or 'elicit emotions' [16].

Within the last ten years the audio community has succeeded at standing up for sonic subtleties and musical details by fighting for level harmonization against the Loudness War [70]. We advocate for the next community move to consist of releasing the gate of toxic masculinity to include the artistry of women and other historically and systemically marginalized groups of studio professionals. We argue that this progress would also positively impact the artistic quality of the work, e.g., in an interview by Fournet, producer Geffen reflected that "many of the male producers she started out with are now working in the industry, but very few of them are dedicated to projects that are really meaningful to them" [71].

\section{TOWARD STRUCTURAL CHANGE}

Building upon intersectional [10]-[14] and microaggressions scholarship [1]-[3], [8], [9] we have sought to offer a macro-level snapshot of experiences of discrimina- 
tion within the commercial recording studio. Our research highlights how heteropatriarchy structures all aspects of the recording studio from experiences of microaggressions and discrimination, to inequalities in terms of pay and credits.

During the course of this research we encountered a critique suggesting that investigating discrimination in audio engineering from the perspective of microaggressions might have the paradoxical effect of 'making things worse,' either by indicating that women and other equity-deserving groups within the field are not 'tough enough' to succeed within the competitive world of audio engineering and music production, or that conducting a survey of this kind reproduces the inequalities that it seeks to document. To this, we can only point to the poor representation of women and other historically and systematically marginalized groups within the industry [4], [6], [7] and ask the question: how could it get worse?

We also encountered the response, 'Look at that famous engineer who is a woman/transgender person/racial minority: they did just fine.' While we recognize and celebrate the achievements of those who have been able to make a name for themselves despite facing the barriers described in this study, we would like to see a future where historically underrepresented groups can thrive in the studio without having to be absolutely exceptional and without the accompanying baggage of trauma that was so eloquently captured in our open-ended responses.

We are currently working on a qualitative study based on the open-ended survey responses that will highlight how marginalized individuals experience toxic workplace cultures in their daily lives, involving experiences of harassment, discrimination, and challenges to expertise, and the outcomes of these experiences, which include both adaptation behaviors and leaving the business. We believe that our research benefited hugely from sociological and psychological perspectives that informed us what is happening in the commercial recording studio. These research results should help design future actions to address the wide range of issues that we have identified. We also hope that this collaborative project will provide a baseline picture of the work climate in the studio and inspire more interdisciplinary studies to examine other audio workplaces such as R\&D laboratories, live events, sound design studios, or video game companies.

\section{ACKNOWLEDGMENT}

For more details, our statistical results are available in full at: https://opus.uleth.ca/bitstream/handle/10133/ 5813/Brooks-additional-document.pdf? sequence $=8 \&$ isAllowed $=\mathrm{y}$

We would like to thank the translators who generously gave their time to ensure the survey was available in 19 languages-Yuval Adler, Areti Andrepolou, Francesco Bigoni, Rut Elliot Blovmquist, Toby Bol, Julia Borelli, Luciane Cardassi, Fei Fei Du, Nicola Giannini, Thomas Görne, Ollie Haller, Ventsislav Ivanov, Paul Jeukendrup, Kieu Kieu, Ausma Lace, Cesar Lamschtein, Haruka Nagata, Aggie Nichols, Senem Pirler, Kasia Płaczkowska,
Carolina Rodriguez, Shulamit Sarid, Soojeong Seo, Maria Sokolova, and Jördis Weilandt; the student volunteers who helped us promote the survey at AES NY 2019-Toby Bol, Mariana Mejia, Ricardo Morejon, Nate Thomas, and Kieran Turner; and all those who helped us promote the survey, including but not limited to Sam Bennett, Larry Crane (Tape Op Magazine), Martha de Francisco, Matt Dempsey, Leslie Gaston-Bird, Thomas Görne, Jan Herbst, Paul Jeukendrup, Mary Mazurek, Eliezer Oubda, Burç Kostem, and Melina Stephan. Thank you to Ilja Frissen for contributing his statistics expertise and to Jodie Asselin, Adam Patrick Bell, Jean-Phillippe Berrou, Tiffany Boulton, Christopher Churchill, James Goddard, Jason Laurendeau, Gülden Özcan, and Jonathan Sterne for their valuable insights into our survey construction and/or analysis. This study was funded by a Partnership Engage Grant from the Social Sciences and Humanities Research Council of Canada (SSHRC) to be conducted in partnership with AES.

\section{REFERENCES}

[1] J. A. Lewis and H. A. Neville, "Construction and Initial Validation of the Gendered Racial Microaggressions Scale for Black Women," J. Counsel. Psych., vol. 62, no. 2, pp. 289-302 (2015). https://doi.org/10.1037/cou0000062.

[2] K. L. Nadal, "Gender Microaggressions: Implications for Mental Health," in M. A. Paludi (Ed.), Feminism and Women's Rights Worldwide, Volume 2: Mental and Physical Health, pp. 155-175 (Praeger, Santa Barbara, CA, 2010).

[3] Y. Yang and D. W. Carroll, "Gendered Microaggressions in Science, Technology, Engineering, and Mathematics," Lead. Res. Ed., vol. 4, pp. 28-45 (2018).

[4] A. P. Bell, "DAW Democracy? The Dearth of Diversity in 'Playing the Studio,"' J. Music Tech. Ed., vol. 8, no. 2, pp. 129-146 (2015 Jul.). https://doi.org/10.1386/ jmte.8.2.129_1.

[5] M. Mathew, J. Grossman, and A. Andreopoulou, "Women in Audio: Contributions and Challenges in Music Technology and Production," presented at the 141st Convention of the Audio Engineering Society (2016 Sep.), paper 9673.

[6] K. Young, M. Lovedee-Turner, J. Brereton, and H. Daffern, "The Impact of Gender on Conference Authorship in Audio Engineering," (2019) https://tibbakoi.github.io/ aesgender/.

[7] S. L. Smith, M. Choueiti, and K. Pieper, "Inclusion in the Recording Studio?Gender and Race/Ethnicity of Artists, Songwriters \& Producers Across 600 Popular Songs From 2012-2017," USC Annenberg (2018 Jan.). http://assets.uscannenberg.org/docs/inclusion-in-the -recording-studio.pdf.

[8] D. W. Sue, Microaggressions in Everyday Life: Race, Gender, and Sexual Orientation (Wiley \& Sons, Hoboken, NJ, 2010).

[9] D. W. Sue, C. M. Capodilupo, G. C. Torino, J. M. Bucceri, A. M. B. Holder, K. L. Nadal, and M. Esquilin, "Racial Microaggressions in Everyday Life: Implications 
for Clinical Practice," Am. Psychol., vol. 62, no. 4, pp. 271-286 (2007 May/Jun.).

[10] K. Crenshaw, "Demarginalizing the Intersection of Race and Sex: A Black Feminist Critique of Antidiscrimination Doctrine, Feminist Theory, and Antiracist Politics [1989]," in Feminist Legal Theory, pp. 139-167 (Routledge, Oxfordshire, UK, 1991), 1st ed. https://doi.org/10.4324/ 9780429500480-5.

[11] P. Hill Collins, Black Feminist Thought: Knowledge, Consciousness, and the Politics of Empowerment, Revised 10th Anniversary Edition (Routledge, Abingdon, UK, 2000).

[12] L. McCall, "The Complexity of Intersectionality," Signs J. Women Culture Soc., vol. 30, no. 3, pp. 1771-1800 (2005).

[13] P. H. Collins, Intersectionality as Critical Social Theory (Duke University Press, Durham, NC, 2019). https://doi.org/10.1515/9781478007098.

[14] J. Misra, C. V. Curington, and V. M. Green, "Methods of Intersectional Research," Sociol. Spec., vol. 41, no. 1, pp. 9-28 (2020 Jul.). https://www.tandfonline.com/doi/full/10.1080/02732173. 2020.1791772 ?casa_token $=6$ SCyc 16 SXkYAAAAA $\%$ 3A30RFU8QoOuo_VvjwFoQ2iLwkSYON6Adu77ghC38 jwez9Hu2pguzJ8X9JzKRogeiBNyBw1Xm2BbY.

[15] A. Pras and C. Guastavino, "The Role of Music Producers and Sound Engineers in the Current Recording Context, as Perceived by Young Professionals," $\mathrm{Mu}$ sicae Scientiae, vol. 15, no. 1, pp. 73-95 (2011 Mar.). https://doi.org/10.1177/1029864910393407.

[16] A. Watson and J. Ward, "Creating the Right 'Vibe': Emotional Labour and Musical Performance in the Recording Studio," Environ. Plan. A, vol. 45, no. 12, pp. 29042918 (2013 Dec.). https://doi.org/10.1068/a45619.

[17] A. R. Hochschild, The Managed Heart: Commercialization of Human Feeling (University of California Press, Berkeley, CA, 2012). https://doi.org/10.1525/ 9780520951853.

[18] H. Massey, Behind the Glass: Top Record Producers Tell How They Craft the Hits (Miller Freeman Books, San Francisco, CA, 2000).

[19] M. Jarrett, "The Self-Effacing Producer: Absence Summons Presence," in S. Frith and S. Zagorski-Thomas (Eds.), The Art of Record Production: An Introductory Reader for a New Academic Field, pp. 128-148 (Routledge, Oxfordshire, UK, 2012), 1st ed. https://doi.org/10.4324/ 9781315612638.

[20] A. Pras, C. Cance, and C. Guastavino, "Record Producers' Best Practices for Artistic Direction-From Light Coaching to Deeper Collaboration With Musicians," $J$. New Music Res., vol. 42, no. 4, pp. 381-395 (2013 Dec.). https://doi.org/10.1080/09298215.2013.848903.

[21] A. Pras, "What Has Been Left Unsaid About Studio Practices: How Producers and Engineers Prepare, Manage, and Direct Recording Sessions," in A. King and E. Himonides (Eds.), Music, Technology, and Education, pp. 27-44 (Routledge, Oxfordshire, UK, 2016), 1st ed.

[22] P. Wolfe, Women in the Studio: Creativity, Control and Gender in Popular Music Production (Rout- ledge, London, UK, 2019), 1st ed. https://doi.org/10.4324/ 9781315546711.

[23] P. Thompson, Creativity in the Recording Studio: Alternative Takes (Palgrave Macmillan, London, UK, 2018). https://doi.org/10.1007/978-3-030-01650-0.

[24] P. W. Peterson, "History of Women in Audio," presented at the 66th Convention of the Audio Engineering Society (1980 May), paper 1625.

[25] L. Gaston-Bird, Women in Audio (Focal Press, Waltham, MA, 2019). https://doi.org/10.4324/ 9780429455940.

[26] S. Walby, Theorizing Patriarchy (Basil Blackwell Limited, Oxford, UK, 1990).

[27] M. Arvin, E. Tuck, and A. Morrill, "Decolonizing Feminism: Challenging Connections Between Settler Colonialism and Heteropatriarchy," Feminist Formations, vol. 25, no. 1, pp. 8-34 (2013). https://doi.org/10.1353/ ff. 2013.0006 .

[28] A. Hennion, "An Intermediary Between Production and Consumption: The Producer of Popular Music," Sci. Technol. Human Values, vol. 14, no. 4, pp. 400-424 (1989 Oct.). https://doi.org/10.1177/ 016224398901400405.

[29] A. K. Fournet, "Women Rockers and the Strategies of a Minority Position," Music Arts Action, vol. 3, no. 1, pp. 20-47 (Éditions des archives contemporaines, Paris, France, 2010).

[30] M. Buscatto "Jazz Criticism: A Man's World," in Gender in Art Criticism/Du Genre dans la Critique d'Art, pp. 17-29 (2017 Jan.).

[31] K. Marie, "Conversations With Women in $\mathrm{Mu}-$ sic Production," in R. Hepworth-Sawyer J. Hodgson and M. Marrington (Eds.), Producing Music, pp. 311328 (Routledge, Oxfordshire, UK, 2019), 1st ed. https:// doi.org/10.4324/9781315212241-19.

[32] H. Marstal, "Slamming the Door to the Recording Studio - Or Leaving It Ajar?" in R. HepworthSawyer J. Hodgson, L. King, and M. Marrington (Eds.), Gender in Music Production, pp. 127-144 (Focal Press, Waltham, MA, 2020), 1st ed. https://doi.org/10.4324/ 9780429464515-10.

[33] R. J. Burgess, "Producer Compensation: Challenges and Options in the New Music Business," J. Art Record Prod., vol. 3, pp. 1-9 (2008 Nov.).

[34] K. Neuenfeldt, "Learning to Listen When There Is Too Much to Hear: Music Producing and Audio Engineering as 'Engaged Hearing,", Media Int. Aus., vol. 123, no. 1, pp. 150-160 (2007 May). https://doi.org/10.1177/ $1329878 \times 0712300114$.

[35] A. Pras, C. Guastavino, and M. Lavoie, "The Impact of Technological Advances on Recording Studio Practices," J. Am. Soc. Inf. Sci. Technol., vol. 64, no. 3, pp. 612-626 (2013 Mar.). https://doi.org/10.1002/asi.22840.

[36] S. Bennett, Modern Records, Maverick Methods Technology and Process in Popular Music Record Production 1978-2000 (Bloomsbury Academic, London, UK, 2019). https://doi.org/10.5040/9781501344138.

[37] B. Sandstrom, "Women Mix Engineers and the Power of Sound," in P. Moisala and B. Diamond (Eds.), 
Music and Gender, pp. 289-305 (University of Illinois Press, Champaign, IL, 2000).

[38] S. S. Horning, "Engineering the Performance: Recording Engineers, Tacit Knowledge and the Art of Controlling Sound," Social Studies Sci., vol. 34, no. 5, pp. 703-731 (2004 Oct.). https://doi.org/10.1177/ 0306312704047536.

[39] A. Pras, K. Turner, T. Bol, and E. Olivier, "Production Processes of Pop Music Arrangers in Bamako, Mali," presented at the 147th Convention of the Audio Engineering Society (2019 Oct.), paper 10296.

[40] J. M. Gander, "Situating Creative Production: Recording Studios and the Making of a Pop Song," Management Decision, vol. 53, no. 4, pp. 843-856 (2015). https://doi.org/10.1108/md-03-2014-0165.

[41] D. Minchella, "The Poetics of Space: The Role and Co-performance of the Spatial Environment in Popular Music Production," in S. Bennett and E. Bates (Eds.), Critical Approaches to the Production of Music and Sound, pp. 41-61 (Bloomsbury, London, UK, 2018). https://doi.org/10.5040/9781501332074. 0009 .

[42] K. Keightley, “'Turn it Down!' She Shrieked: Gender, Domestic Space, and High Fidelity," Рориlar Music, vol. 15, no. 2, pp. 149-177 (1996 May). https://doi.org/10.1017/s0261143000008096.

[43] C. Pierce, "Offensive Mechanisms," in F. B. Barbour (Ed.), The Black Seventies, pp. 265-282 (Porter Sargent, Boston, MA, 1970).

[44] D. W. Sue, D. Sue, H. A. Neville, and L. Smith, Counseling the Culturally Diverse: Theory and Practice (John Wiley \& Sons, Hoboken, NJ, 2019), 8th ed.

[45] K. Charmaz, Constructing Grounded Theory: A Practical Guide Through Qualitative Analysis (SAGE, Thousand Oaks, CA, 2006), 1st ed.

[46] C. Labuski and C. Keo-Meier, "The (Mis)Measure of Trans," Trans. Studies Quarter., vol. 2, no. 1, pp. 13-33 (2015 Feb.).

[47] J. J. Cameron and D. A. Stinson, "Gender (Mis)Measurement: Guidelines for Respecting Gender Diversity in Psychological Research," Social Pers. Psych. Compass, vol. 13, no. 11, e12506 (2019 Nov.).

[48] G. Norman, "Likert Scales, Levels of Measurement and the "Laws" of Statistics," Adv. Health Sci. Ed., vol. 15, no. 5, pp. 625-632 (2010). https://doi.org/10.1007/ s10459-010-9222-y.

[49] J. Cohen, Statistical Power Analysis for the Behavioral Sciences, 2nd ed. (L. Erlbaum Associates, Hillsdale, NJ, 1988). https://doi.org/10.1016/b978-0-12-179060-8. 50005-0.

[50] J. Cohen, “A Power Primer," Psych. Bulletin, vol. 112, no. 1, pp. 155-159 (1992). https://doi.org/10.1037/ 0033-2909.112.1.155.

[51] G. Corbie-Smith, S. B. Thomas, and D. M. M. St. George, "Distrust, Race, and Research," Arch. Intern. Med., vol. 162, no. 21, pp. 2458-2463 (2002 Nov.). https://doi.org/10.1001/archinte.162.21.2458.

[52] T. Zuberi and E. Bonilla-Silva (Eds.), White Logic,
White Methods: Racism and Methodology (Rowman \& Littlefield Publishers, Inc., Lanham, MD, 2008).

[53] M. Kovach, Indigenous Methodologies: Characteristics, Conversations, and Contexts (University of Toronto Press, Toronto, Canada, 2010).

[54] L. T. Smith, Decolonizing Methodologies: Research and Indigenous Peoples (Zed Books Limited, Auckland, New Zealand, 2013).

[55] U. R. Hülsheger and A. F. Schewe, "On the Costs and Benefits of Emotional Labor: A Meta-Analysis of Three Decades of Research," J. Occup. Health Psych., vol. 16, no. 3, pp. 361-389 (2011). https://doi.org/10.1037/a0022876.

[56] V. -Y. Koon and P. -Y. Pun, "The Mediating Role of Emotional Exhaustion and Job Satisfaction on the Relationship Between Job Demands and Instigated Workplace Incivility," J. Appl. Behav. Sci., vol. 54, no. 2, pp. 187-207 (2018 Jun.). https://doi.org/10.1177/ 0021886317749163.

[57] G. Blau and L. Andersson, "Testing a Measure of Instigated Workplace Incivility," J. Occup. Organiz. Psych., vol. 78, no. 4, pp. 595-614 (2005 Dec.). https://doi.org/10.1348/096317905x26822.

[58] D. D. van Jaarsveld, D. D. Walker, and D. P. Skarlicki, "The Role of Job Demands and Emotional Exhaustion in the Relationship Between Customer and Employee Incivility," J. Manage., vol. 36, no. 6, pp. 1486-1504 (2010 Nov.). https://doi.org/10.1177/0149206310368998.

[59] J. E. Bartlett II, M. E. Bartlett, and T. G. Reio, Jr., "Workplace Incivility: Worker and Organizational Antecedents and Outcomes," in Proceedings of the Academy of Human Resource Development International Research Conference in the Americas (Panama City, FL) (2008 Feb.).

[60] A. H. Eagly, "The His and Hers of Prosocial Behavior: An Examination of the Social Psychology of Gender," Am. Psych., vol. 64, no. 8, pp. 644-658 (2009). https://doi.org/10.1037/e669392012-012.

[61] T. A. Kupers, "Toxic Masculinity as a Barrier to Mental Health Treatment in Prison," J. Clin. Psych., vol. 61, no. 6, pp. 713-724 (2005 Jun.). https://doi.org/10.1002/ jclp.20105.

[62] J. L. Berdahl, M. Cooper, P. Glick, R. W. Livingston, and J. C. Williams, "Work as a Masculinity Contest," J. Social Issues, vol. 74, no. 3, pp. 422-448 (2018 Sep.). https://doi.org/10.1111/josi.12289.

[63] B. Derks, C. Van Laar, and N. Ellemers, "The Queen Bee Phenomenon: Why Women Leaders Distance Themselves From Junior Women," Leader. Quarter., vol. 27, no. 3, pp. 456-469 (2016 Jun.). https://doi.org/ 10.1016/j.leaqua.2015.12.007.

[64] B. Derks, N. Ellemers, C. van Laar, and K. de Groot, "Do Sexist Organizational Cultures Create the Queen Bee?" Brit. J. Social Psych., vol. 50, no. 3, pp. 519-535 (2011 Sep.). https://doi.org/10.1348/014466610x525280.

[65] S. A. M. Buckingham and M. Ronan, "Factors Contributing to Gender Imbalance in the Audio Industry," presented at the 146th Convention of the Audio Engineering Society (2019 Mar.), paper 10159.

[66] B. Prior, E. Barra, and S. Kramer, "Women in the US Music Industry" (2020). http:// 
datasim.info/wp-content/uploads/2019/04/Women-in-the -U.S.-Music-Industry-Report.pdf.

[67] M. Duguid, "Female Tokens in High-Prestige Work Groups: Catalysts or Inhibitors of Group Diversification?" Organiz. Behav. Human Decision Process., vol. 116, no. 1, pp. 104-115 (2011 Sep.). https://doi.org/10.1016/ j.obhdp.2011.05.009.

[68] O. Marshall, "Shibboleths in the Studio: Informal Demarcation Practices Among Audio Engineers," Social
Studies Sci., vol. 50, no. 6, pp. 881-900 (2020 Dec.). https://doi.org/10.1177/0306312720922314.

[69] F. E. Toole, "Audio - Science in the Service of Art," The Audio Critic, vol. 28, pp. 5-14 (2002).

[70] F. Camerer, "On the Way to Loudness Nirvana Audio Levelling With EBU R 128," EBU Tech. Rev. (2010).

[71] A. Fournet, Women Music Producers: Sonic Innovation From the Periphery of a Male-Dominated Industry, Ph.D. thesis, New York University, NY (2019).

\section{A.1: Additional Tables and Figures}

Table A1. P-values for TAS analysis, color-coded by effect size. No large or medium effect sizes were found. Entries in light gray/yellow indicate small effect size (Cramer's V > 0.07 or 0.1 ). Non-significant entries are in white with gray text. Slope entry color indicates direction of relationship of dependent variable with AGE (light gray/pink positive, dark gray/blue negative).

\begin{tabular}{|c|c|c|c|c|c|c|c|c|c|c|}
\hline \multirow[t]{3}{*}{ 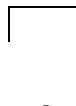 } & & \multirow{3}{*}{$\mathbf{N}$} & GEN & SOR & RET & DIS & MIG & GNI & \multicolumn{2}{|c|}{ AGE } \\
\hline & & & \multicolumn{6}{|c|}{$\mathbf{p}\left(\chi^{2}\right)$} & $\mathbf{P}(\mathbf{t})$ & Slope \\
\hline & & & \multicolumn{8}{|c|}{ ALL AGES } \\
\hline \multirow{15}{*}{ 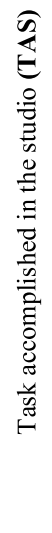 } & Tracking & \multirow{7}{*}{$\underset{\infty}{\infty}$} & 0.25 & 0.07 & 0.15 & 0.09 & 0.83 & 0.94 & 0.06 & -0.02 \\
\hline & Editing & & 0.43 & 0.18 & 0.96 & 0.08 & 0.19 & 0.09 & 0.07 & -0.02 \\
\hline & Mixing & & 0.70 & $9.68 \mathrm{E}-3$ & 0.20 & 0.01 & 0.12 & 0.98 & 0.02 & -0.03 \\
\hline & Mastering & & $2.09 \mathrm{E}-3$ & $3.67 \mathrm{E}-3$ & 0.35 & 0.21 & 0.67 & 0.90 & 0.02 & 0.02 \\
\hline & Producing & & 0.34 & 0.05 & 0.34 & 0.28 & 0.12 & 0.77 & 0.84 & 0.00 \\
\hline & Assisting & & $2.06 \mathrm{E}-5$ & $3.05 \mathrm{E}-3$ & 0.05 & 0.73 & 0.95 & 0.56 & $8.16 \mathrm{E}-19$ & -0.13 \\
\hline & Other & & 0.52 & 0.36 & 0.95 & $4.49 \mathrm{E}-04$ & 0.90 & 0.01 & 0.93 & 0.00 \\
\hline & & \multirow{8}{*}{ 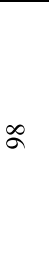 } & \multicolumn{8}{|c|}{$\mathrm{AGE}<30$} \\
\hline & Tracking & & 0.75 & 0.04 & & 0.01 & & & & \\
\hline & Editing & & 0.33 & 0.32 & & 0.38 & & & & \\
\hline & Mixing & & 0.96 & 0.02 & & 0.42 & & & & \\
\hline & Mastering & & 0.04 & 0.01 & & 0.12 & & & & \\
\hline & Producing & & 0.30 & 0.01 & & 0.36 & & & & \\
\hline & Assisting & & 0.09 & 0.01 & & 0.64 & & & & \\
\hline & Other & & 0.24 & 0.38 & & $2.23 \mathrm{E}-03$ & & & & \\
\hline
\end{tabular}

Table A2. Dunn's post-hoc test findings for one-way Kruskal-Wallis tests on aggregate microaggressions scores. $\delta$ is equal to the score for demographic category 2 minus the score for demographic category 1 - so for example, the $\delta$ value reported for CM/CW indicates the score for cisgender women minus the score for cisgender men. For Likert-scale questions, a $\delta$ of 1 indicates a difference of one point. For DRA, $\delta>0$ indicates more often being properly credited. For microaggressions factors, $\delta>0$ indicates more often experiencing microaggressions from that factor. For AESM, $\delta>0$ indicates more often being an AES member. For AESD, $\delta>0$ indicates more often being aware of the AES D\&I Committee. Significant differences are in gray, non-significant differences are in white with gray text. Abbreviations: $\mathrm{CM}=$ cisgender man; $\mathrm{CW}=$ cisgender woman; $\mathrm{TNB}=$ trans/non binary; $\mathrm{H}=$ heterosexual; $\mathrm{NH}=$ non-heterosexual; $\mathrm{RN}=$ Either white, or BIPOC and not part of a racial/ethnic minority in place of work; RY=Both BIPOC and part of a racial/ethnic minority in place of work; $\mathrm{ND}=$ non-disabled; $\mathrm{ID}=$ invisible disability; $\mathrm{VD}=$ visible disability; $\mathrm{MN}=$ lives in country of birth; $\mathrm{MY}=$ lives in country other than country of birth; $\mathrm{L}=$ low/lower middle income country; $\mathrm{H}=$ upper middle/high income country.

\begin{tabular}{|c|c|c|c|c|c|c|c|c|c|c|c|c|}
\hline & \multirow[b]{2}{*}{ UNIT } & \multirow[b]{2}{*}{$\mathbf{N}$} & \multicolumn{3}{|c|}{ GEN } & \multirow{2}{*}{$\begin{array}{c}\text { SOR } \\
\delta: \\
\text { H/NH }\end{array}$} & \multirow{2}{*}{$\begin{array}{c}\text { RET } \\
\delta: \\
\text { RN/RY }\end{array}$} & \multicolumn{3}{|c|}{ DIS } & \multirow{2}{*}{$\begin{array}{c}\text { MIG } \\
\delta: \\
\text { NM/MY }\end{array}$} & \multirow{2}{*}{$\begin{array}{c}\text { GNI } \\
\delta: \\
\text { L/H }\end{array}$} \\
\hline & & & $\begin{array}{c}\delta: \\
\mathrm{CM} / \\
\mathrm{CW}\end{array}$ & $\begin{array}{c}\delta: \\
\mathrm{CM} / \\
\mathrm{TNB}\end{array}$ & $\begin{array}{c}\delta: \\
\mathrm{CW} / \\
\mathrm{TNB}\end{array}$ & & & $\begin{array}{c}\delta: \\
\mathrm{ND} / \mathrm{ID}\end{array}$ & $\begin{array}{c}\delta: \\
\text { ND/VD }\end{array}$ & $\begin{array}{c}\delta: \\
\text { ID/VD }\end{array}$ & & \\
\hline & USD & 138 & -58.60 & -49.40 & 9.20 & 19.24 & -66.21 & -61.47 & 71.77 & 133.24 & 3.46 & 45.35 \\
\hline & LS1 & \multirow{3}{*}{387} & -0.68 & -0.49 & 0.19 & -0.18 & -0.65 & -0.91 & -0.36 & 0.54 & -0.45 & 0.08 \\
\hline AESM & $\mathrm{N}->\mathrm{Y}$ & & 0.16 & 0.05 & -0.11 & -0.06 & 0,15 & 0.26 & 0.26 & 0.00 & 0.10 & 0.33 \\
\hline AESD & $\mathrm{N}->\mathrm{Y}$ & & -0.25 & -0.17 & 0.08 & -0.23 & -0.19 & 0.50 & -0.29 & -0.79 & 0.25 & 0.64 \\
\hline SOB & \multirow[b]{9}{*}{ LS2 } & 283 & 1.14 & 1.23 & 0.08 & 0.50 & 0.34 & 1.14 & 0.52 & -0.62 & -0.02 & -0.18 \\
\hline SAM & & 284 & 1.04 & 1.04 & 0.00 & 0.31 & 0.51 & 0.96 & 0.42 & -0.54 & 0.13 & -0.33 \\
\hline STE & & 281 & 0.82 & 1.01 & 0.18 & 0.17 & 0.20 & 0.79 & -0.10 & -0.90 & 0.07 & -0.67 \\
\hline MGEN & & \multirow{6}{*}{234} & 2.30 & 1.61 & -0.69 & 0.92 & 0.87 & 1.27 & 0.50 & -0.77 & 0.03 & 0.17 \\
\hline MAGE & & & 1.05 & 1.03 & -0.01 & 0.43 & 0.12 & 1.06 & 0.34 & -0.72 & -0.02 & -0.39 \\
\hline MSOR & & & 0.42 & 1.02 & 0.60 & 0.82 & 0.22 & 0.78 & 0.57 & -0.20 & 0.05 & 0.02 \\
\hline MCUL & & & 0.16 & 0.58 & 0.42 & -0.02 & 0.47 & 1.12 & 0.20 & -0.93 & 0.52 & -0.67 \\
\hline MDIS & & & 0.61 & 0.76 & 0.15 & 0.37 & 0.38 & 1.57 & 0.95 & -0.63 & 0.01 & -0.21 \\
\hline MRET & & & 0.11 & 0.42 & 0.32 & 0.01 & 0.88 & 0.94 & 0.24 & -0.70 & 0.21 & -0.72 \\
\hline
\end{tabular}


Table A3. P-values for $\boldsymbol{f}$-statistics from 2-way ANOVA of GEN/SOR for intersection analysis, color coded by effect size. Entries with background in dark gray/red indicate large effect size $\left(\eta^{2}>=0.1379\right)$, entries in medium gray/orange indicate medium effect size ( $\eta^{2}$ $>=0.0588)$, entries in light gray/yellow indicate small effect size $\left(\eta^{2}>=0.0099\right)$. Non-significant entries are in white with gray text.

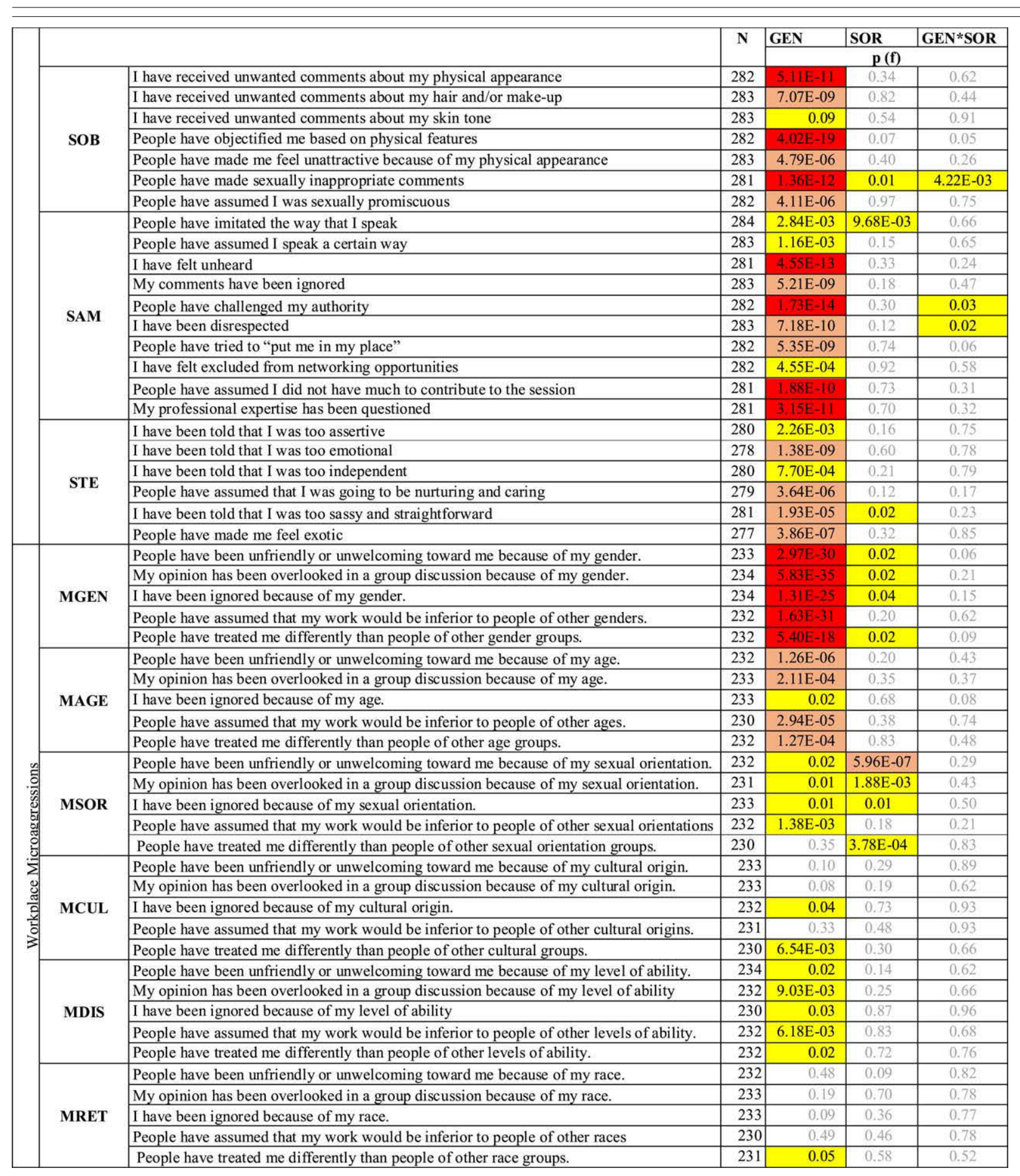



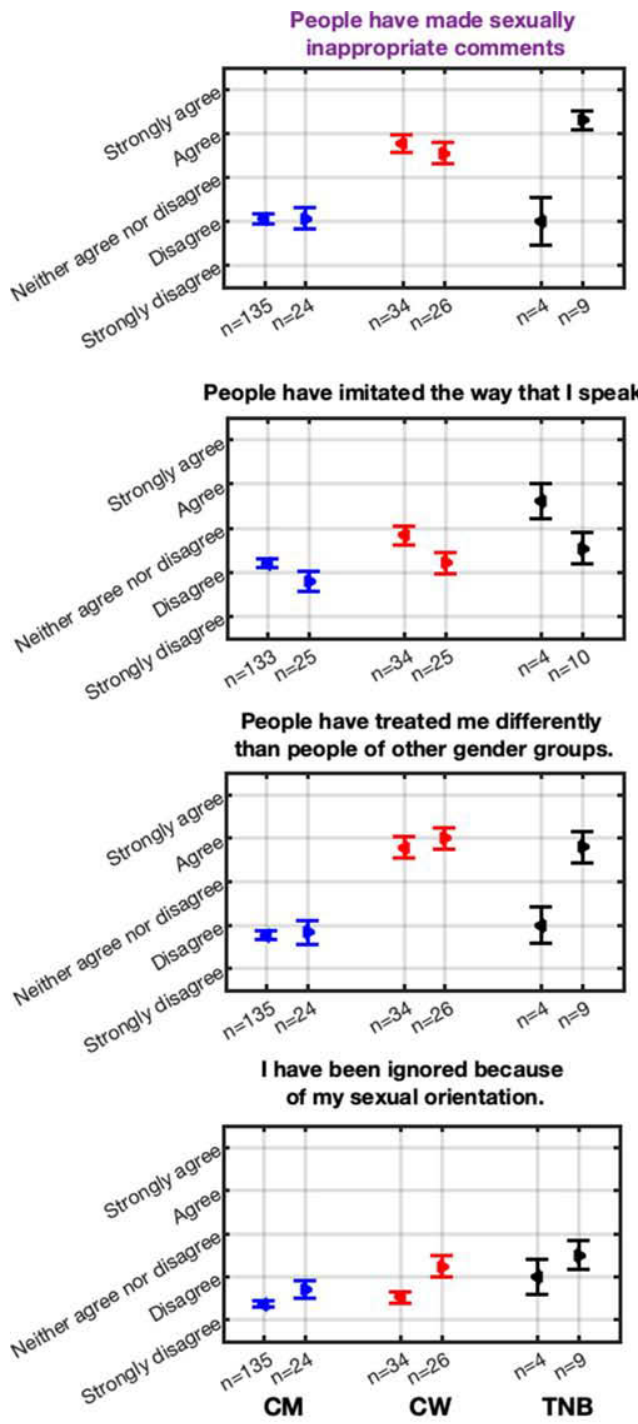

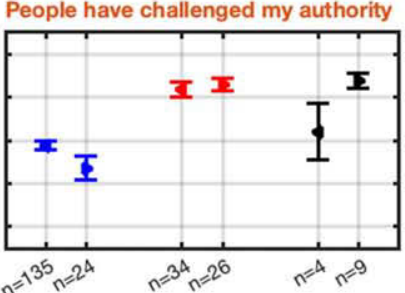

I have been told that I was too sassy and straightforward

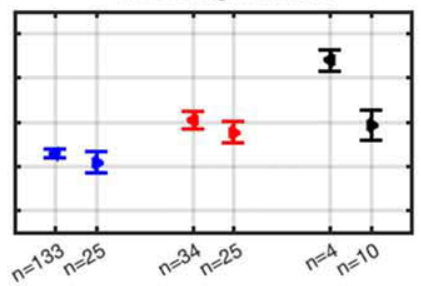

My opinion has been overlooked in a group discussion because of my gender.

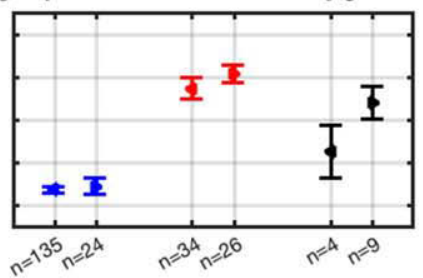

People have been unfriendly or unwelcoming toward me because of my sexual orientation.

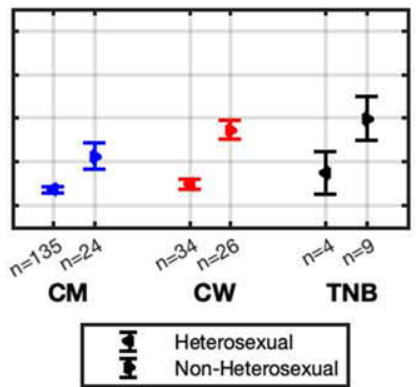

I have been disrespected

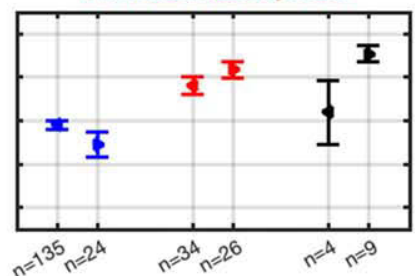

People have been unfriendly or unwelcoming toward me because of my gender.

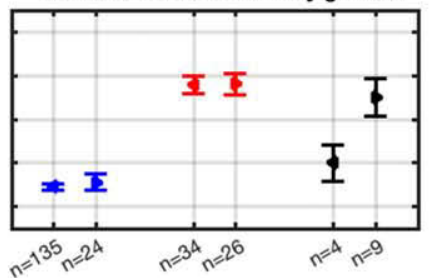

I have been ignored because of my gender.

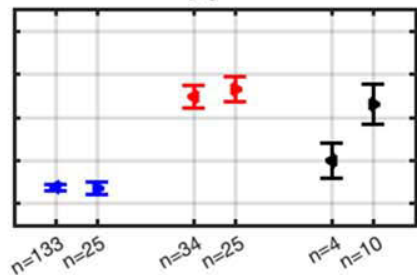

My opinion has been overlooked in a group discussion because of my sexual orientation.

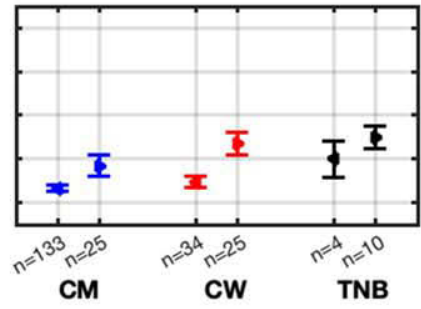

Fig. A1. Microaggressions with a simultaneous effect for GEN/SOR (black titles) and/or a GEN $*$ SOR intersection term (dark gray/purple indicates both simultaneous effect and interaction term; light gray/orange indicates only intersection term).
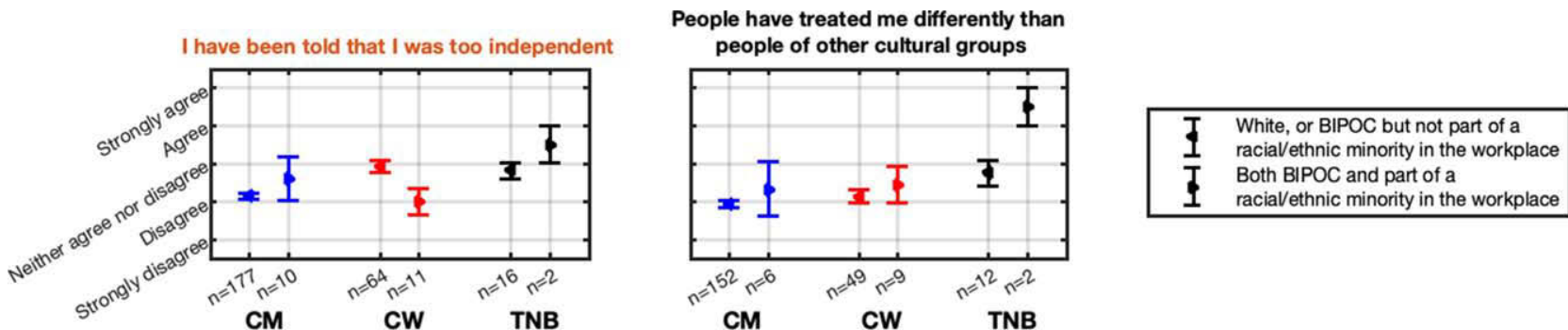

Fig. A2. Microaggressions with a simultaneous effect for GEN/RET (black title) or a GEN*RET intersection term (light gray/orange title). 


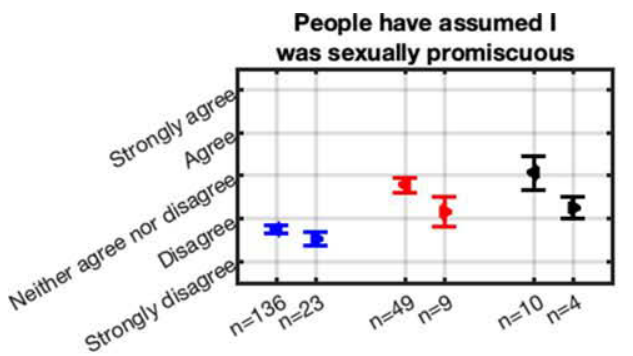

In the My opinion has been overlooked in a group discussion because of my cultural origin.

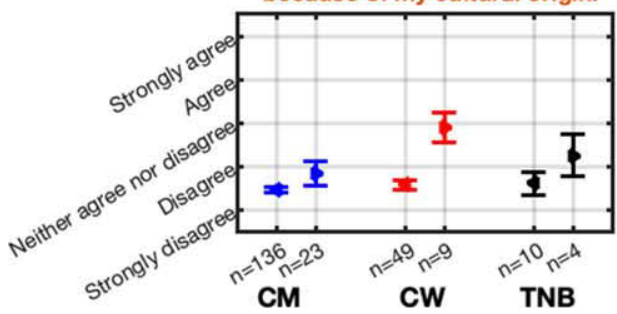

People have made me feel exotic

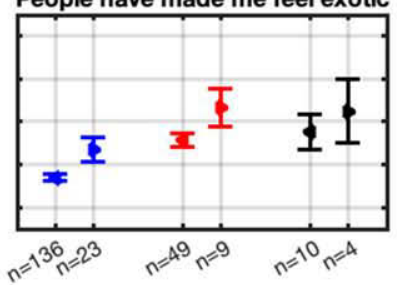

1 Currently living in country of birth

$\overline{\boldsymbol{T}}$ Not currently living in country of birth work would be inferior to people of other cultural origins.

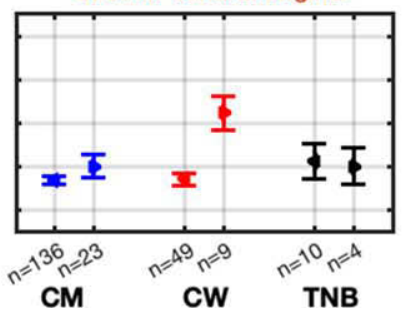

People have been unfriendly or unwelcoming toward me because of my cultural origin.

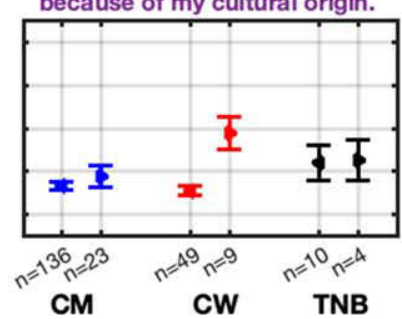

Fig. A3. Microaggressions with a simultaneous effect for GEN/MIG (black titles) and/or a GEN*MIG intersection term (dark gray/purple indicates both simultaneous effect and interaction term; light gray/orange indicates only intersection term). 

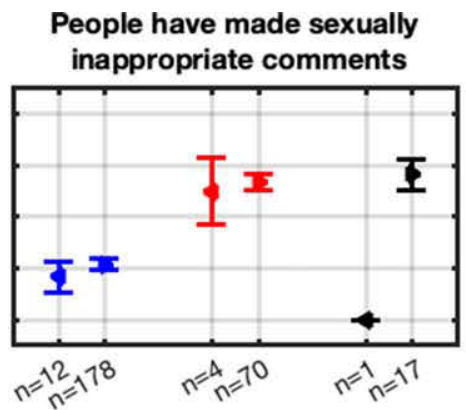

I have been disrespected

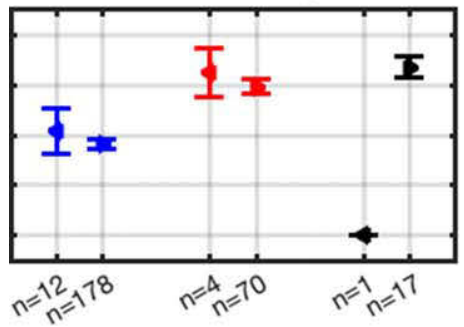

My opinion has been overlooked in a group discussion because of my sexual orientation.

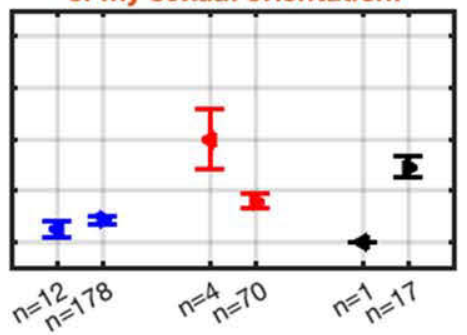

I have been ignored because of my race.

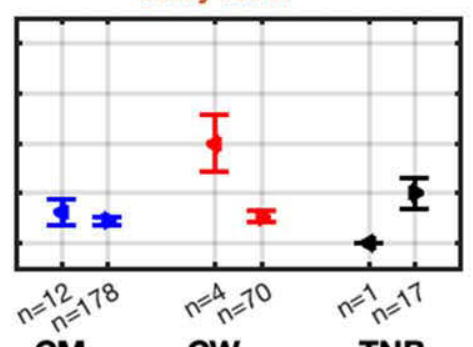

People have imitated the way that I speak

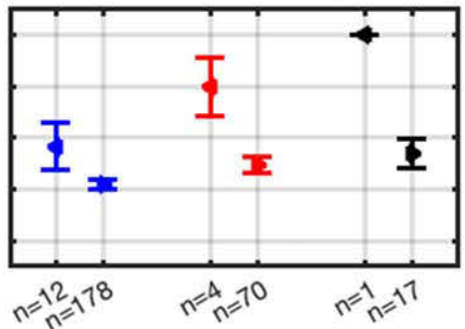

People have treated me differently than people of other cultural groups.

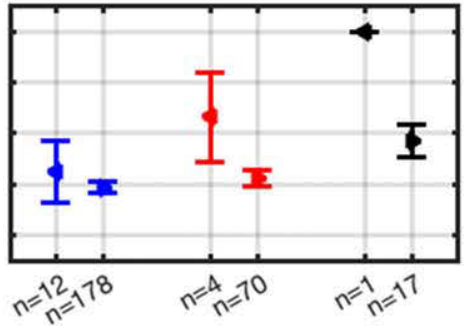

People have been unfriendly or unwelcoming toward me because of my level of ability.

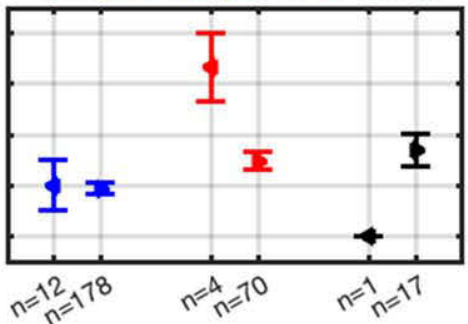

People have treated me differently than people of other racial groups.

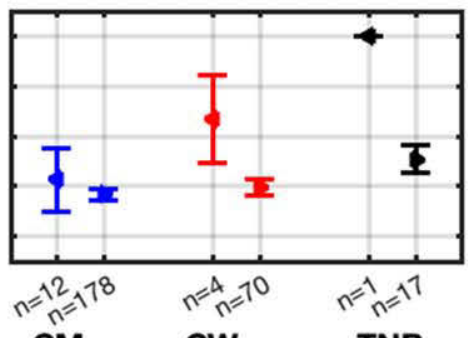

People have challenged my authority

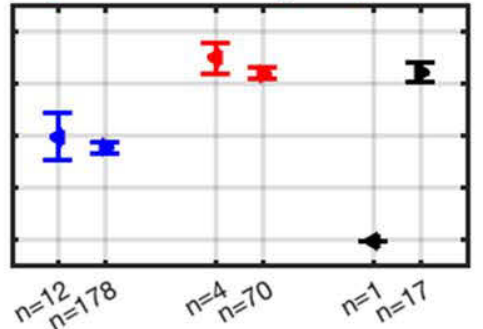

I have been ignored because of my cultural origin.

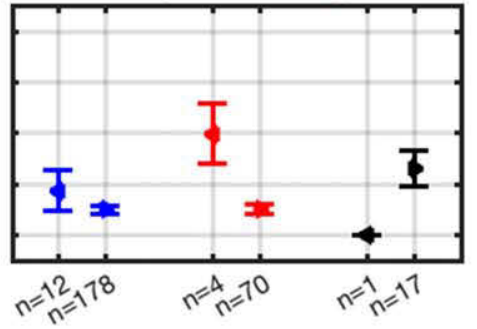

People have assumed that my work would be inferior to people of other levels of ability.

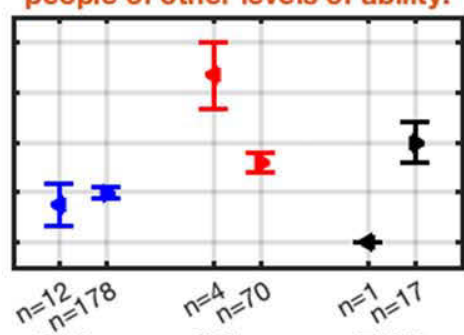

CM CW

TNB

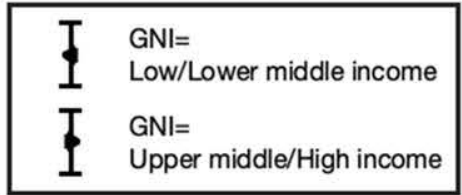

Fig. A4. Microaggressions with a simultaneous effect for GEN/GNI (black titles) or a GEN*GNI intersection term (light gray/orange titles). No microaggression had both a simultaneous effect and an intersection term for GNI. 


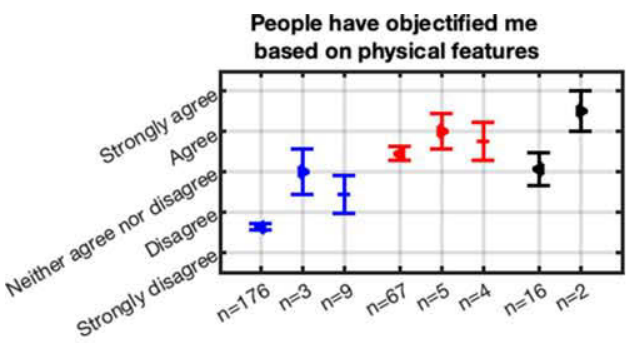

I have received unwanted comments about my physical appearance

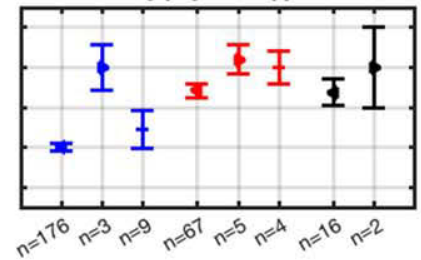

People have tried to "put me in my place"
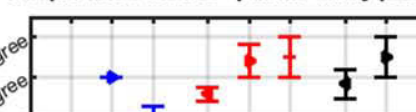

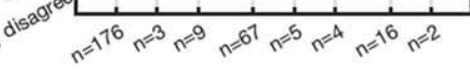

People have been unfriendly or unwelcoming toward me because of my cultural origin

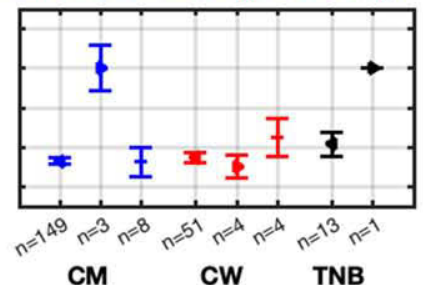

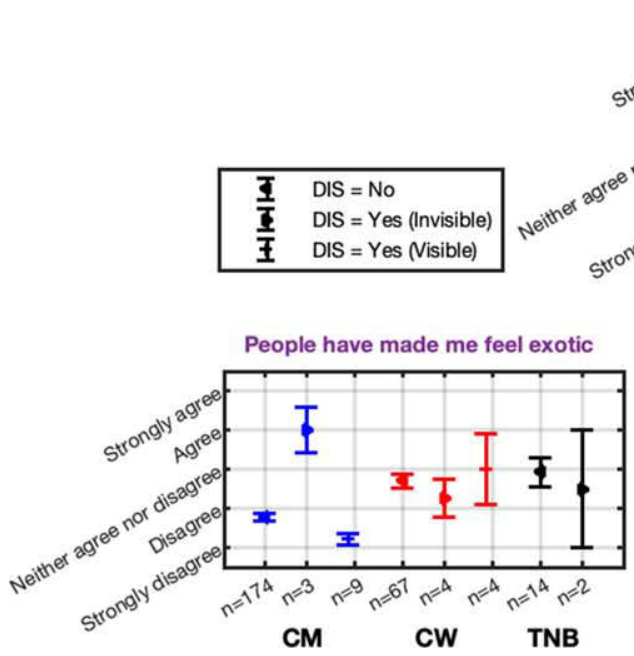

I have received unwanted comments about my hair and/or make-up

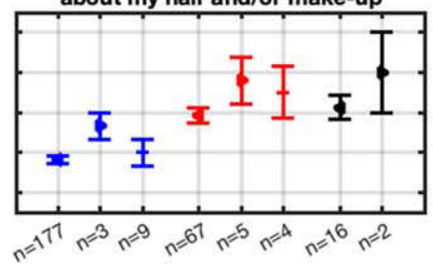

Fig. A5. Microaggressions with a simultaneous effect for GEN/DIS (black titles) and/or a GEN*DIS intersection term (dark gray/purple indicates both simultaneous effect and interaction term; light gray/orange indicates only intersection term). Note that no trans/non-binary participants identified as having a visible disability. 

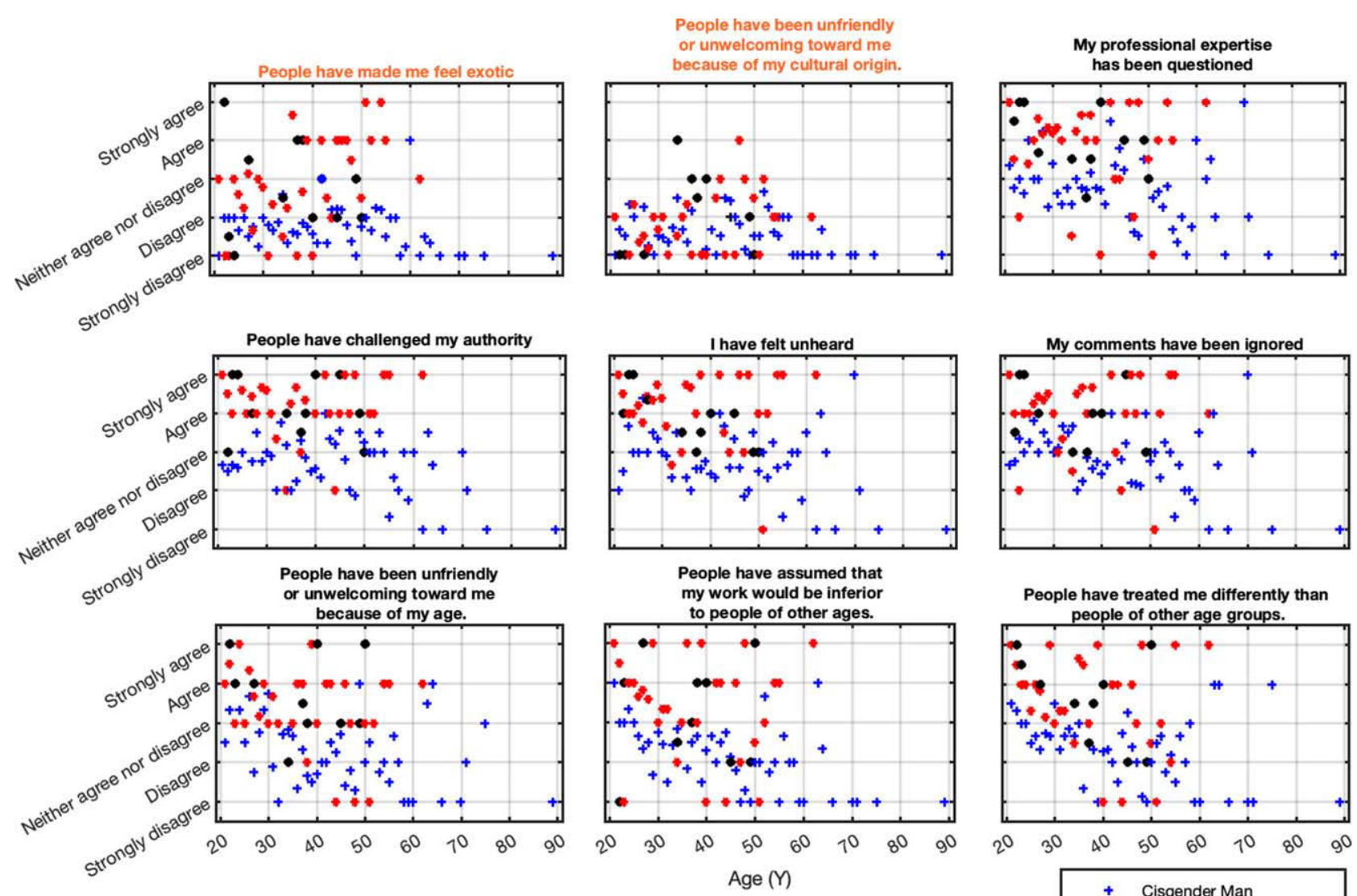

People have treated me differently than people of other age groups.

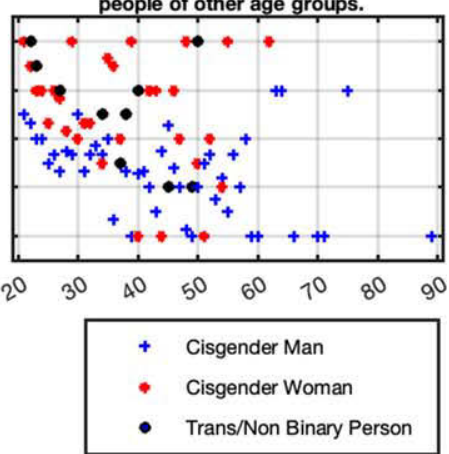

Fig. A6. Microaggressions with a simultaneous effect of GEN/AGE (black titles) or a GEN*AGE intersection term (gray/orange titles). No microaggression had both a simultaneous effect and an intersection term for AGE. Markers represent the mean response for a given gender at a given age, e.g., at age 50, a single cross represents the mean score for cisgender men of age 50, a star for cisgender women of age 50 , and a dot for trans/non-binary people of age 50 . 


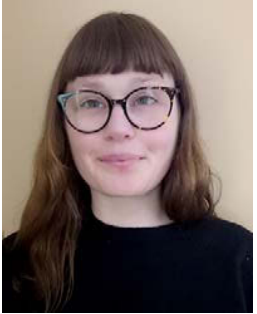

Grace Brooks

\section{THE AUTHORS}

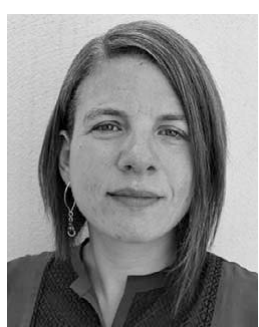

Amandine Pras

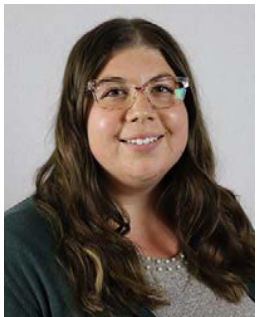

Monica Lockett
Grace Brooks is an experimental musician, live sound engineer, and $\mathrm{PhD}$ candidate in the School of Information Studies at McGill University. They are conducting an interdisciplinary research project linking tacit knowledge and gender performativity in professional practice.

In addition to this project about Gender in the Studio, Amandine Pras is leading The West African Audio Network funded by SSHRC, in partnership with AFRINUM directed by Emmanuelle Olivier and funded by ANR. Her research explores, from a global perspective, how the democratization of digital audio technology that disrupted large-format analog studios and the Western business model of the recording industry has generated extended music performance techniques and innovative audio practices. She completed her $\mathrm{PhD}$ thesis in Information Sciences at McGill on best practices for musical recording in the digital era. Since her graduation from the Paris Conservatoire and her participation in the Audio Recording Engineer Practicum at the Banff Centre, she has pursued a career as a freelance music producer and audio engineer. She has released albums with musicians as diverse as the ARC Ensemble, Jim Black, Quatuor Bozzini, Luciane Cardassi, Daniel Carter, Benoit Delbecq, Mary Halvorson, Tony Malaby, the Metropolis Ensemble, Andy Milne, Satoshi Takeishi, and William Parker.

Dr. Athena Elafros is a qualitative cultural sociologist. She is Assistant Professor of Sociology at the University of Lethbridge.

Monica Lockett is an undergraduate student in Sociology at the University of Lethbridge. A journalist by trade, she received a diploma in Communication Arts from Lethbridge College and has worked in northern Alberta as a radio news reporter and anchor. Her research experience and interests focus on the intersections of protest, journalism, and political duty; how journalists conceive of their professional role and identity; and analyzing qualitative experiences of microaggression and discrimination in audio recording studios. 